\title{
CROSS PRODUCTS, INVARIANTS, AND CENTRALIZERS
}

\author{
GEORGIA BENKART* AND ALBERTO ELDUQUE*
}

Dedicated to Efim Zelmanov on the occasion of his 60 th birthday.

\begin{abstract}
An algebra $V$ with a cross product $\times$ has dimension 3 or 7. In this work, we use 3-tangles to describe, and provide a basis for, the space of homomorphisms from $\mathrm{V}^{\otimes n}$ to $\mathrm{V}^{\otimes m}$ that are invariant under the action of the automorphism group $\operatorname{Aut}(\mathrm{V}, \times)$ of $\mathrm{V}$, which is a special orthogonal group when $\operatorname{dim} \mathrm{V}=3$, and a simple algebraic group of type $\mathrm{G}_{2}$ when $\operatorname{dim} \mathrm{V}=7$. When $m=n$, this gives a graphical description of the centralizer algebra $\operatorname{End}_{\text {Aut }(\mathrm{V}, \times)}\left(\mathrm{V}^{\otimes n}\right)$, and therefore, also a graphical realization of the Aut $(\mathrm{V}, \times)$-invariants in $\mathrm{V}^{\otimes 2 n}$ equivalent to the First Fundamental Theorem of Invariant Theory. We show how the 3-dimensional simple Kaplansky Jordan superalgebra can be interpreted as a cross product (super)algebra and use 3-tangles to obtain a graphical description of the centralizers and invariants of the Kaplansky superalgebra relative to the action of the special orthosymplectic group.
\end{abstract}

\section{INTRODUCTION}

A cross product algebra $(\mathrm{V}, \mathrm{b}, \times)$ is a finite-dimensional vector space $\mathrm{V}$ over a field $\mathbb{F}$ (assumed to have characteristic different from 2 ) with a nondegenerate symmetric bilinear form $\mathrm{b}$ and a bilinear multiplication $\mathrm{V} \times \mathrm{V} \rightarrow \mathrm{V}$, $(x, y) \mapsto x \times y$, that satisfies

$$
\begin{aligned}
& \mathrm{b}(x \times y, x)=0, \\
& x \times x=0, \\
& \mathrm{~b}(x \times y, x \times y)=\mathrm{b}(x, x) \mathrm{b}(y, y)-\mathrm{b}(x, y) \mathrm{b}(y, x),
\end{aligned}
$$

for any $x, y \in \mathrm{V}$. Nonzero cross products exist only if $\operatorname{dim}_{\mathbb{F}} \mathrm{V}=3$ or 7 (see [BG67], Ros04]), and when $\mathbb{F}$ is the field of real numbers, the cross product is the familiar one from calculus in dimension 3 if $b$ is positive definite. We relate cross product algebras to certain 3-tangle categories and give a graphical realization of the invariants and centralizer algebras of tensor powers of $\mathrm{V}$ under the action of its automorphism group Aut $(\mathrm{V}, \times)$.

The 3-tangle category $\mathcal{T}$ has as objects the finite sets $[n]=\{1,2, \ldots, n\}$ for $n \in \mathbb{N}=\{0,1,2, \ldots\}$, where $[0]=\emptyset$. For any $n, m \in \mathbb{N}$, the morphisms

2010 Mathematics Subject Classification. Primary 20G05, 17B10.

Key words and phrases. Cross product; invariant map; 3-tangle; $\mathrm{G}_{2}$; Kaplansky superalgebra; centralizer algebra.

* Both authors have been supported by the Spanish Ministerio de Economía y Competitividad and Fondo Europeo de Desarrollo Regional (FEDER) MTM 2013-45588-C3-2-P. The second author also acknowledges support by the Diputación General de Aragón Fondo Social Europeo (Grupo de Investigación de Álgebra). He is also grateful for the hospitality of the Department of Mathematics of the University of Wisconsin-Madison in November 2015. Most of the results of this paper were obtained during that visit. 
$\operatorname{Mor}_{\mathcal{T}}([n],[m])$ are $\mathbb{F}$-linear combinations of 3-tangles, and they are generated through composition and disjoint union from the basic morphisms (basic 3tangles) in (3.6) and (3.7). This gives a graphical calculus that enables us to describe the space $\operatorname{Hom}_{\text {Aut }(\mathrm{V}, \times)}\left(\mathrm{V}^{\otimes n}, \mathrm{~V}^{\otimes m}\right)$ of Aut $(\mathrm{V}, \times)$-homomorphisms on tensor powers of $\mathrm{V}$. When $\operatorname{dim}_{\mathbb{F}} \mathrm{V}=7$, the group Aut $(\mathrm{V}, \times)$ is a simple algebraic group of type $G_{2}$, and $V$ is its natural 7-dimensional module (its smallest nontrivial irreducible module). When $\operatorname{dim}_{\mathbb{F}} \mathrm{V}=3$, Aut $(\mathrm{V}, \times)$ is the special orthogonal group $\mathrm{SO}(\mathrm{V}, \mathrm{b})$.

From the properties of the cross product, we construct three homomorphisms (when $\operatorname{dim}_{\mathbb{F}} \mathrm{V}=7$, they are given in (4.6), (4.3), and when $\operatorname{dim}_{\mathbb{F}} \mathrm{V}=$ 3, in (5.5),(5.2) ). Applying methods similar to those in (Kup94, Kup96, [CKR05]), we show in each case (see Theorems 4.10 and 5.12 for the precise statements) that these homomorphisms correspond to a set $\Gamma^{*}$ consisting of three 3 -tangle relations, and the following result holds. In the statement, the 3 -tangles must satisfy some additional constraints. When $\operatorname{dim}_{\mathbb{F}} \mathrm{V}=3$, these constraints are incorporated in the definition of "normalized" 3-tangles.

Theorem 1.1. Let $n, m \in \mathbb{N}$ and assume that the characteristic of $\mathbb{F}$ is 0 . Let $(\mathrm{V}, \mathrm{b}, \mathrm{\times})$ be a vector space $\mathrm{V}$ endowed with a nonzero cross product $x \times y$ relative to the nondegenerate symmetric bilinear form $\mathrm{b}$.

(a) The classes modulo $\Gamma^{*}$ of (normalized) 3-tangles $[n] \rightarrow[m]$ without crossings and without any of the subgraphs in (4.13) form a basis of $\operatorname{Mor}_{\Gamma_{\Gamma^{*}}}([n],[m])$.

(b) There is a functor $\mathcal{R}_{\Gamma^{*}}$ giving a linear isomorphism

$$
\operatorname{Mor}_{\mathcal{T}_{\Gamma^{*}}}([n],[m]) \rightarrow \operatorname{Hom}_{\text {Aut }(\mathrm{V}, \times)}\left(\mathrm{V}^{\otimes n}, \mathrm{~V}^{\otimes m}\right) .
$$

(c) The 3-tangles $[n] \rightarrow[n]$ as in part (a) give a basis of the centralizer algebra

$$
\operatorname{End}_{\text {Aut }(\mathrm{V}, \times)}\left(\mathrm{V}^{\otimes n}\right) \simeq \operatorname{Mor}_{\mathcal{T}_{\Gamma^{*}}}([n],[n]) .
$$

The centralizer algebra $\operatorname{End}_{\mathrm{Aut}(\mathrm{V}, \times)}\left(\mathrm{V}^{\otimes n}\right)$ is isomorphic to the space of Aut $(\mathrm{V}, \times)$-invariants in $\mathrm{V}^{\otimes 2 n}$, and part (c) gives a graphical realization of that space equivalent to the First Fundamental Theorem of Invariant Theory results in ([Wey39, [Schw88, [ZSh15]).

Section 2 establishes basic facts about bilinear forms on tensor powers and Section 3 about 3-tangles. In Sections 4 and 5 , we prove the above theorem for dimensions 7 and 3 respectively. The final section extends these considerations to the Kaplansky superalgebra. This 3-dimensional simple Jordan superalgebra has a multiplication that can be interpreted as a cross product relative to a nondegenerate supersymmetric bilinear form $\mathrm{b}$. With modifications to the arguments that take into account the fact that the cross product is supercommutative, $x \times y=(-1)^{x y} y \times x$, and so depends on the parity of the homogeneous elements $x, y$, we are able in Theorem 6.7 to prove an analogue of Theorem 1.1, where the automorphism group Aut $(\mathrm{V}, \times)$ is the special orthosymplectic group $\operatorname{SOSp}(\mathrm{V}, \mathrm{b})$ (or equivalently, the group could be replaced by the orthosymplectic Lie superalgebra $\mathfrak{o s p}(\mathrm{V}, \mathrm{b})$ of derivations on $\mathrm{V}$ ). This gives a graphical version of the First Fundamental Theorem of Invariant Theory for $\operatorname{SOSp}(\mathrm{V}, \mathrm{b})$ (see [Ser92, [LZ15]).

In all the 3-dimensional cases, the centralizer algebra $\operatorname{End}_{\mathrm{Aut}(\mathrm{V}, \times)}\left(\mathrm{V}^{\otimes n}\right)$ has dimension equal to the number $a(2 n)$ of Catalan partitions i.e., partitions 
of $2 n$ points on a circle into a nonintersecting union of subsets, each of size at least 2, whose convex hulls are disjoint (sequence \#A099251 in [OEIS]).

The cross product and certain properties that it satisfies are key to deriving the 3-tangle results in this paper. This approach differs from that of $\mathrm{Ku}$ perberg Kup94, which derives quantum $\mathrm{G}_{2}$ link invariants from the Jones polynomial starting from its simplest formulation in terms of the Kauffman bracket. Kuperberg's quantum $\mathrm{G}_{2}$ invariant is defined not only on knots and links, but also on regular isotopy classes of certain graphs with endpoints and all other nodes trivalent, akin to the 3-tangles here, and is a particular case of the Reshetikhin-Turaev invariant of ribbon graphs.

\section{Preliminaries}

Let $\mathrm{V}$ be a finite-dimensional nonzero vector space over a field $\mathbb{F}$ endowed with a nondegenerate bilinear form $\mathrm{b}: \mathrm{V} \times \mathrm{V} \rightarrow \mathbb{F}$, which we will identify with the linear map $\mathrm{b}: \mathrm{V}^{\otimes 2} \rightarrow \mathbb{F}$ given by $\mathrm{b}(u \otimes v)=\mathrm{b}(u, v)$. For $n \in \mathbb{N}=$ $\{0,1,2, \ldots\}$, consider the linear map

$$
\begin{gathered}
\mathrm{b}_{n}: \mathrm{V}^{\otimes 2 n} \longrightarrow \mathbb{F} \\
v_{1} \otimes \cdots \otimes v_{2 n} \mapsto \prod_{i=1}^{n} \mathrm{~b}\left(v_{i}, v_{2 n+1-i}\right),
\end{gathered}
$$

with $\mathrm{b}_{0}=$ id (the identity map on $\mathrm{V}^{\otimes 0}:=\mathbb{F}$ ). For $n \geq 1$, $\mathrm{b}_{n}$ will be represented graphically as follows:

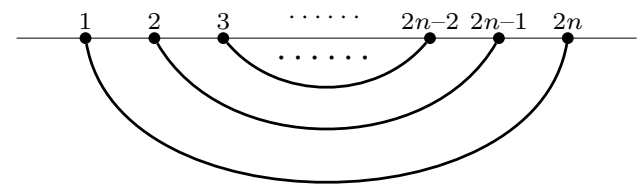

where no crossings appear. (This will be important later on.)

Also, $\mathrm{b}_{n}$ will be identified with the nondegenerate bilinear form $\mathrm{V}^{\otimes n} \times \mathrm{V}^{\otimes n} \rightarrow \mathbb{F}$ such that

$$
\mathrm{b}_{n}\left(v_{1} \otimes \cdots \otimes v_{n}, w_{1} \otimes \cdots w_{n}\right)=\prod_{i=1}^{n} \mathrm{~b}\left(v_{i}, w_{n+1-i}\right),
$$

for any $v_{1}, \ldots, v_{n}, w_{1}, \ldots, w_{n} \in \mathrm{V}$. If $\mathbf{b}$ is symmetric, so is $\mathbf{b}_{n}$ for any $n \geq 1$.

Definition 2.1. Given $n, m \in \mathbb{N}$ and a linear map $f \in \mathrm{Hom}_{\mathbb{F}}\left(\mathrm{V}^{\otimes n}, \mathrm{~V}^{\otimes m}\right)$, the transpose of $f$ is the linear map $f^{t} \in \mathrm{Hom}_{\mathbb{F}}\left(\mathrm{V}^{\otimes m}, \mathrm{~V}^{\otimes n}\right)$ such that

$$
\mathbf{b}_{m}(f(x), y)=\mathbf{b}_{n}\left(x, f^{t}(y)\right),
$$

for any $x \in \mathrm{V}^{\otimes n}$ and $y \in \mathrm{V}^{\otimes m}$.

In other words,

$$
\mathrm{b}_{m} \circ\left(f \otimes \mathrm{id}_{m}\right)=\mathrm{b}_{n} \circ\left(\mathrm{id}_{n} \otimes f^{t}\right)
$$

in $\mathrm{Hom}_{\mathbb{F}}\left(\mathrm{V}^{\otimes(n+m)}, \mathbb{F}\right)$, where $\mathrm{id}_{n}$ denotes the identity map on $\mathbf{V}^{\otimes n}$. 
Equation (2.2) will be represented graphically as follows:

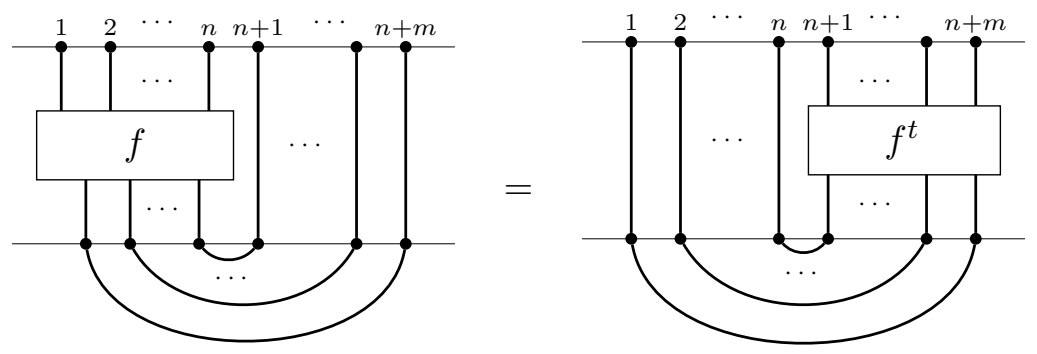

Given linear maps $f \in \mathrm{Hom}_{\mathbb{F}}\left(\mathrm{V}^{\otimes n}, \mathrm{~V}^{\otimes q}\right), g \in \mathrm{Hom}_{\mathbb{F}}\left(\mathrm{V}^{\otimes m}, \mathrm{~V}^{\otimes p}\right)$, the linear map $f \otimes g \in \mathrm{Hom}_{\mathbb{F}}\left(\mathrm{V}^{\otimes(n+m)}, \mathrm{V} \otimes(q+p)\right.$ will be represented as follows:

The next result is clear.

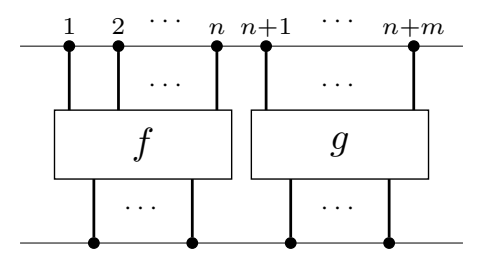

\section{Proposition 2.3.}

- $\left(\mathrm{id}_{n}\right)^{t}=\mathrm{id}_{n}$ for any $n \in \mathbb{N}$.

- $(f \circ g)^{t}=g^{t} \circ f^{t}$, for any $n, m, p \in \mathbb{N}, f \in \operatorname{Hom}_{\mathbb{F}}\left(\mathrm{V}^{\otimes m}, \mathrm{~V}^{\otimes p}\right)$, and $g \in \mathrm{Hom}_{\mathbb{F}}\left(\mathrm{V}^{\otimes n}, \mathrm{~V}^{\otimes m}\right)$.

- $(f \otimes g)^{t}=g^{t} \otimes f^{t}$ for any $n, m, p, q \in \mathbb{N}, f \in \mathrm{Hom}_{\mathbb{F}}\left(\mathrm{V}^{\otimes n}, \mathrm{~V}^{\otimes q}\right)$, and $g \in \mathrm{Hom}_{\mathbb{F}}\left(\mathrm{V}^{\otimes m}, \mathrm{~V}^{\otimes p}\right)$.

- If $\mathrm{b}$ is symmetric, $\left(f^{t}\right)^{t}=f$ for any $n \in \mathbb{N}$ and $f \in \mathrm{Hom}_{\mathbb{F}}\left(\mathrm{V}^{\otimes n}, \mathrm{~V}^{\otimes m}\right)$.

Assume $\operatorname{dim}_{\mathbb{F}} \mathrm{V}=d$, and let $\left\{u_{i}\right\}_{i=1}^{d}$ and $\left\{v_{i}\right\}_{i=1}^{d}$ be dual bases relative to $\mathrm{b}$ so that $\mathrm{b}\left(u_{i}, v_{j}\right)=\delta_{i, j}$ (1 if $i=j$ and 0 otherwise).

For any $n \in \mathbb{N}$, let $[d]^{n}:=\{1, \ldots, d\}^{n}\left([d]^{0}=\{\emptyset\}\right)$, and for any $\alpha=$ $\left(\alpha_{1}, \ldots, \alpha_{n}\right) \in[d]^{n}$, let $\bar{\alpha}:=\left(\alpha_{n}, \ldots, \alpha_{1}\right), u_{\alpha}:=u_{\alpha_{1}} \otimes \cdots \otimes u_{\alpha_{n}}$, and $v_{\alpha}:=v_{\alpha_{1}} \otimes \cdots \otimes v_{\alpha_{n}}$. For $n=0$, set $u_{\emptyset}=1=v_{\emptyset}$ and $\bar{\emptyset}=\emptyset$. Then $\left\{u_{\alpha}\right\}_{\alpha \in[d]^{n}}$ and $\left\{v_{\bar{\alpha}}\right\}_{\alpha \in[d]^{n}}$ are dual bases of $\mathrm{V}^{\otimes n}$ relative to $\mathrm{b}_{n}$. Hence, for any $x \in \mathrm{V}^{\otimes n}$,

$$
x=\sum_{\alpha \in[d]^{n}} \mathrm{~b}_{n}\left(u_{\alpha} \otimes x\right) v_{\bar{\alpha}}=\sum_{\alpha \in[d]^{n}} \mathrm{~b}_{n}\left(x \otimes v_{\bar{\alpha}}\right) u_{\alpha} .
$$

For any $n, m \in \mathbb{N}, f \in \operatorname{Hom}_{\mathbb{F}}\left(\mathrm{V}^{\otimes n}, \mathrm{~V}^{\otimes m}\right)$, and $\alpha \in[d]^{n}, f\left(u_{\alpha}\right)=\sum_{\beta \in[d]^{m}} a_{\alpha, \beta} u_{\beta}$, with $a_{\alpha, \beta} \in \mathbb{F}$ for any $\beta$. Then, for any $\beta \in[d]^{m}$ :

$$
f^{t}\left(v_{\bar{\beta}}\right)=\sum_{\alpha \in[d]^{n}} a_{\alpha, \beta} v_{\bar{\alpha}}
$$

because

$$
a_{\alpha, \beta}=\mathrm{b}_{m}\left(f\left(u_{\alpha}\right) \otimes v_{\bar{\beta}}\right)=\mathrm{b}_{n}\left(u_{\alpha} \otimes f^{t}\left(v_{\bar{\beta}}\right)\right) .
$$

\section{Proposition 2.5.}

(i) For any $n \in \mathbb{N}, \mathrm{b}_{n}^{t}(1)=\sum_{\alpha \in[d]^{n}} v_{\bar{\alpha}} \otimes u_{\alpha}$. 
(ii) For any $n, m \in \mathbb{N}$, the linear map

$$
\begin{aligned}
\Phi_{n, m}: \operatorname{Hom}_{\mathbb{F}}\left(\mathrm{V}^{\otimes n}, \mathrm{~V}^{\otimes m}\right) & \longrightarrow \operatorname{Hom}_{\mathbb{F}}\left(\mathrm{V}^{\otimes(n+m)}, \mathbb{F}\right) \\
f & \mapsto \mathrm{b}_{m} \circ\left(f \otimes \mathrm{id}_{m}\right),
\end{aligned}
$$

is a linear isomorphism with inverse given by

$$
\begin{aligned}
\Psi_{n, m}: \operatorname{Hom}_{\mathbb{F}}\left(\mathrm{V}^{\otimes(n+m)}, \mathbb{F}\right) & \longrightarrow \mathrm{Hom}_{\mathbb{F}}\left(\mathrm{V}^{\otimes n}, \mathrm{~V}^{\otimes m}\right) \\
h & \mapsto\left(h \otimes \mathrm{id}_{m}\right) \circ\left(\mathrm{id}_{n} \otimes \mathrm{b}_{m}^{t}\right) .
\end{aligned}
$$

(iii) For any $n, m \in \mathbb{N}$ and $f \in \operatorname{Hom}_{\mathbb{F}}\left(\mathrm{V}^{\otimes n}, \mathrm{~V}^{\otimes m}\right)$,

$$
f^{t}=\left(\mathrm{id}_{n} \otimes \mathrm{b}_{m}\right) \circ\left(\mathrm{id}_{n} \otimes f \otimes \mathrm{id}_{m}\right) \circ\left(\mathrm{b}_{n}^{t} \otimes \mathrm{id}_{m}\right) .
$$

Proof. For (i), we must prove that $\mathrm{b}_{n}(x \otimes y)=\mathrm{b}_{2 n}\left(x \otimes y \otimes \mathrm{b}_{n}^{t}(1)\right)$ for any $x, y \in \mathrm{V}^{\otimes n}$. But

$$
\begin{aligned}
\mathrm{b}_{n}(x \otimes y) & =\mathrm{b}_{n}\left(x \otimes\left(\sum_{\alpha \in[d]^{n}} \mathrm{~b}_{n}\left(y \otimes v_{\bar{\alpha}}\right) u_{\alpha}\right)\right) \\
& =\sum_{\alpha \in[d]^{n}} \mathrm{~b}_{n}\left(x \otimes u_{\alpha}\right) \mathrm{b}_{n}\left(y \otimes v_{\bar{\alpha}}\right) \\
& =\mathrm{b}_{2 n}\left(x \otimes y \otimes\left(\sum_{\alpha \in[d]^{n}} v_{\bar{\alpha}} \otimes u_{\alpha}\right)\right) .
\end{aligned}
$$

(ii) Now, for $n, m \in \mathbb{N}$ and $f \in \operatorname{Hom}_{\mathbb{F}}\left(\mathrm{V}^{\otimes n}, \mathrm{~V}^{\otimes m}\right)$,

$$
\left(\left(\mathrm{b}_{m} \circ\left(f \otimes \mathrm{id}_{m}\right)\right) \otimes \mathrm{id}_{m}\right) \circ\left(\mathrm{id}_{n} \otimes \mathrm{b}_{m}^{t}\right)=\left(\mathrm{b}_{m} \otimes \mathrm{id}_{m}\right) \circ\left(f \otimes \mathrm{b}_{m}^{t}\right),
$$

and for any $x \in \mathrm{V}^{\otimes n}$,

$$
x \stackrel{f \otimes \mathrm{b}_{m}^{t}}{\longmapsto} \sum_{\alpha \in[d]^{m}} f(x) \otimes v_{\bar{\alpha}} \otimes u_{\alpha} \stackrel{\mathrm{b}_{m} \otimes \mathrm{id}_{m}}{\longmapsto} \sum_{\alpha \in[d]^{m}} \mathrm{~b}_{m}\left(f(x) \otimes v_{\bar{\alpha}}\right) u_{\alpha}=f(x) .
$$

Also, for $h \in \operatorname{Hom}_{\mathbb{F}}\left(\mathrm{V}^{\otimes(n+m)}, \mathbb{F}\right)$,

$\mathrm{b}_{m} \circ\left(\left(\left(h \otimes \mathrm{id}_{m}\right) \circ\left(\mathrm{id}_{n} \otimes \mathrm{b}_{m}^{t}\right)\right) \otimes \mathrm{id}_{m}\right)=\mathrm{b}_{m} \circ\left(h \otimes \mathrm{id}_{m} \otimes \mathrm{id}_{m}\right) \circ\left(\mathrm{id}_{n} \otimes \mathrm{b}_{m}^{t} \otimes \mathrm{id}_{m}\right)$, and for $x \in \mathrm{V}^{\otimes n}$ and $y \in \mathrm{V}^{\otimes m}$,

$$
\begin{aligned}
& x \otimes y \stackrel{\mathrm{id}_{n} \otimes \mathbf{b}_{m}^{t} \otimes \mathrm{id}_{m}}{\longmapsto} \sum_{\alpha \in[d]^{n}} x \otimes v_{\bar{\alpha}} \otimes u_{\alpha} \otimes y \stackrel{h \otimes \mathrm{id}_{m} \otimes \mathbf{i d}_{m}}{\longmapsto} \sum_{\alpha \in[d]^{m}} h\left(x \otimes v_{\bar{\alpha}}\right) u_{\alpha} \otimes y \\
& \stackrel{\mathrm{b}_{m}}{\longmapsto} \sum_{\alpha \in[d]^{n}} h\left(x \otimes v_{\bar{\alpha}}\right) \mathrm{b}_{m}\left(u_{\alpha} \otimes y\right)=\sum_{\alpha \in[d]^{m}} h\left(x \otimes \mathrm{b}_{m}\left(u_{\alpha} \otimes y\right) v_{\bar{\alpha}}\right)=h(x \otimes y) .
\end{aligned}
$$

(iii) Finally, for $n, m \in \mathbb{N}, f \in \operatorname{Hom}_{\mathbb{F}}\left(\mathbf{V}^{\otimes n}, \mathbf{V}^{\otimes m}\right)$, and $x \in \mathrm{V}^{\otimes m}$,

$$
\begin{aligned}
& x \stackrel{\mathrm{b}_{m}^{t} \otimes \mathrm{id}_{m}}{\longmapsto} \sum_{\alpha \in[d]^{n}} v_{\bar{\alpha}} \otimes u_{\alpha} \otimes x \stackrel{\mathrm{id}_{n} \otimes f \otimes \mathrm{id}_{m}}{\longmapsto} \sum_{\alpha \in[d]^{n}} v_{\bar{\alpha}} \otimes f\left(u_{\alpha}\right) \otimes x \\
& \stackrel{\operatorname{id}_{n} \otimes \mathbf{b}_{m}}{\longmapsto} \sum_{\alpha \in[d]^{n}} \mathrm{~b}_{m}\left(f\left(u_{\alpha}\right) \otimes x\right) v_{\bar{\alpha}}=\sum_{\alpha \in[d]^{n}} \mathrm{~b}_{n}\left(u_{\alpha} \otimes f^{t}(x)\right) v_{\bar{\alpha}}=f^{t}(x) .
\end{aligned}
$$


The map $\mathrm{b}_{n}^{t}: \mathrm{V}^{\otimes 0}=\mathbb{F} \mapsto \mathrm{V}^{\otimes 2 n}$ will be represented as follows:

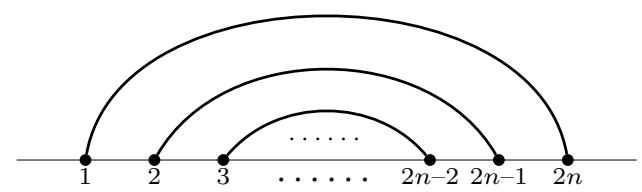

Then $\Phi_{n, m}$ corresponds to:
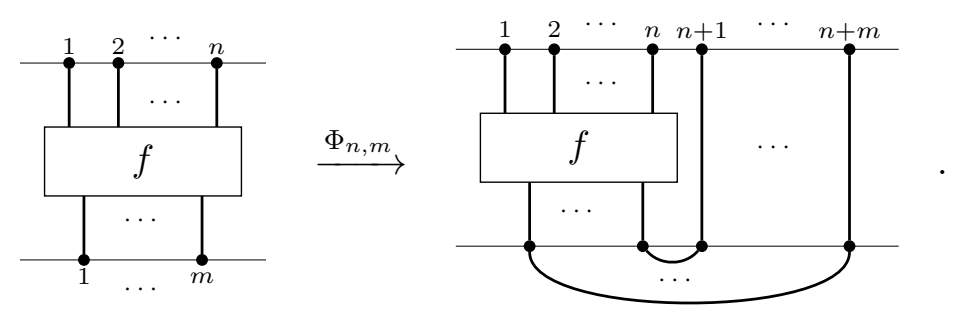

and $\Psi_{n, m}$ to
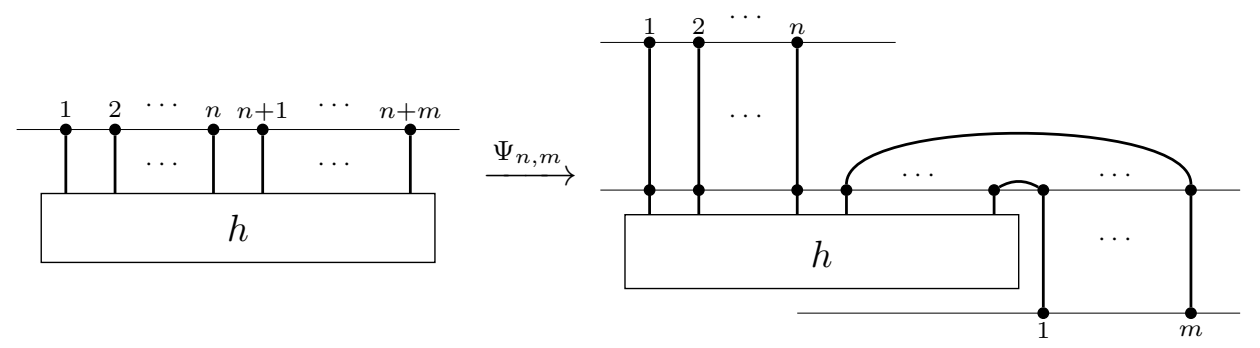

$(2.7)$

Lastly, the result in item (iii) is represented as follows:
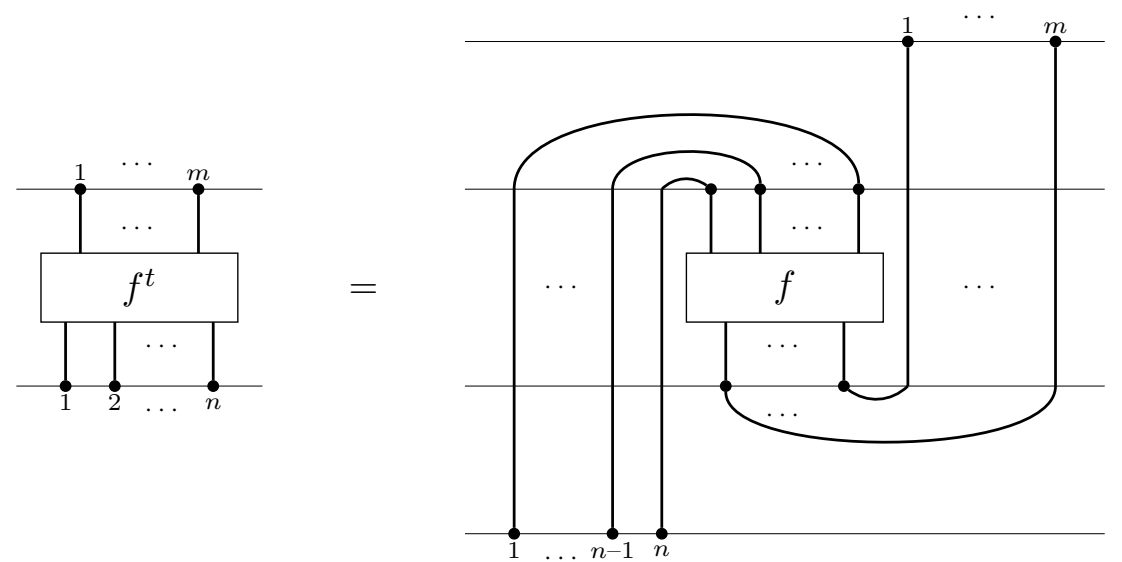

Remark 2.9. From item (i) in Proposition 2.5 we get:

$$
\left(\mathbf{b} \circ \mathbf{b}^{t}\right)(1)=\sum_{i=1}^{d} \mathrm{~b}\left(v_{i} \otimes u_{i}\right)= \begin{cases}\operatorname{dim}_{\mathbb{F}} \mathrm{V} & \text { if } \mathrm{b} \text { is symmetric, } \\ -\operatorname{dim}_{\mathbb{F}} \mathrm{V} & \text { if } \mathrm{b} \text { is skew symmetric. }\end{cases}
$$




\section{3-TANGLES}

In this section, we establish the facts needed about 3-tangles and certain 3tangle categories, following for the most part the definitions and conventions of [CKR05], but with some slight modifications.

3.1. 3-tangles, composition and disjoint union. Let $G=(V, E)$ be a finite, undirected graph, where $V$ is the set of nodes (vertices) and $E$ is the set of edges, which is a subset of the set of unordered pairs $u v$ of different nodes, $u, v \in V, u \neq v$.

The valency of a node $v$ is the number $\operatorname{val}(v)$ of edges incident with $v$. The cyclic group $C_{3}$ acts on the Cartesian power $E^{3}$ by cyclic permutations. Denote by $E^{3} / C_{3}$ the set of orbits, and by $\left[e_{1}, e_{2}, e_{3}\right]$ the orbit of $\left(e_{1}, e_{2}, e_{3}\right)$.

We will consider only graphs $G=(V, E)$ with $1 \leq \operatorname{val}(v) \leq 3$ for all $v \in V$, and which are endowed with a map (orientation)

$$
\nu:\{v \in V: \operatorname{val}(v)=3\} \rightarrow E^{3} / C_{3},
$$

such that, for any $v \in V$ with $\operatorname{val}(v)=3, \nu(v)=\left[e_{1} e_{2} e_{3}\right]$, where $e_{1}, e_{2}, e_{3}$ are the edges incident with $\nu$. That is, $\nu$ chooses one of the two possible orientations of the edges incident with $v$. Orientations are indicated by a coloring, black for positive (clockwise) and white for negative (counterclockwise):

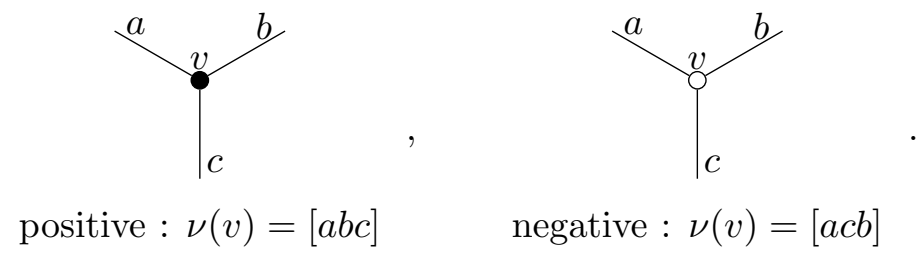

The set of nodes of valency 1 will be called the boundary of $G$ and denoted by $\partial G$. A path in $G=(V, E)$ is a subgraph $P$ with nodes $\left\{v_{0}, v_{1}, \ldots, v_{k}\right\}$ and edges $\left\{v_{0} v_{1}, v_{1} v_{2}, \ldots, v_{k-1} v_{k}\right\}$, where the $v_{i}$ 's are pairwise distinct. We write $P=v_{0} v_{1} \cdots v_{k}$ and call $k$ the length of $P$. A refinement of a graph $G=(V, E)$ is a graph obtained from $G$ by dividing some of the edges, i.e., by replacing these edges with paths having the same endpoints and so that intermediate nodes are not nodes of $G$, and different edges involve different new nodes:

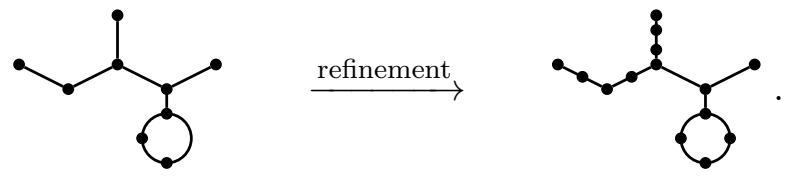

Two such graphs $G=(V, E)$ and $G^{\prime}=\left(V^{\prime}, E^{\prime}\right)$ are said to be equivalent if $\partial G=\partial G^{\prime}$, and they admit identical refinements. Each equivalence class has a representative having no nodes of valency 2 except for the nodes on circles.

For any $n \in \mathbb{N}$, let $[n]=\{1, \ldots, n\}$ with $[0]=\emptyset$. Then, for $n, m \in \mathbb{N}$, a 3tangle $\gamma:[n] \rightarrow[m]$ is an equivalence class of graphs $G=(V, E)$ with $\partial G=$ $[n] \sqcup[m]$ (disjoint union, which may thought of as $\left\{1, \ldots, n, 1^{\prime}, \ldots, m^{\prime}\right\}$ ). This is represented graphically by taking a representative with no nodes of valency 2 (except for the circles), drawn between two lines, as in the next 
example $\gamma:[7] \rightarrow[4]$

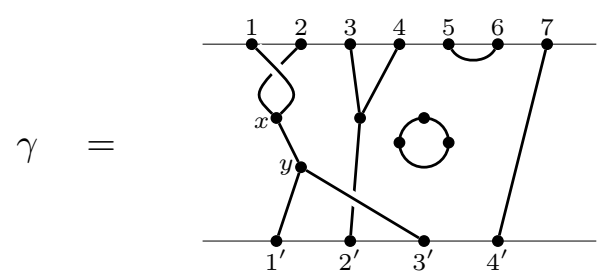

where the orientation of a valency 3 node (like the node labeled by $x$ in (3.1)) is given by clockwise order: $\nu(x)=[2 x 1 x y x]$, that is, we have:

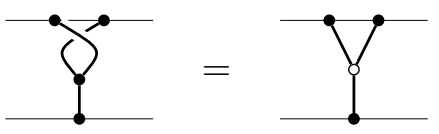

and hence only positively oriented nodes will be used. Moreover, we will usually suppress the $\bullet$ 's in our figures:

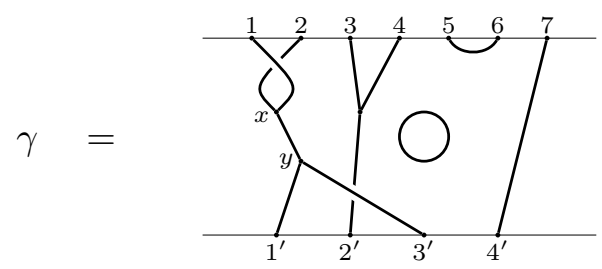

The disjoint union of two 3-tangles $\gamma:[n] \rightarrow[m]$ and $\gamma^{*}:\left[n^{*}\right] \rightarrow\left[m^{*}\right]$ is the 3-tangle $\gamma \sqcup \gamma^{*}:\left[n+n^{*}\right] \rightarrow\left[m+m^{*}\right]$ obtained by juxtaposing $\gamma^{*}$ to the right of $\gamma$ and shifting the numbering on $\gamma$ as illustrated below. The composition of two 3-tangles $\gamma:[n] \rightarrow[m]$ and $\gamma^{*}:[m] \rightarrow[r]$ is the tangle $\gamma^{*} \circ \gamma:[n] \rightarrow[r]$ obtained by bordism:
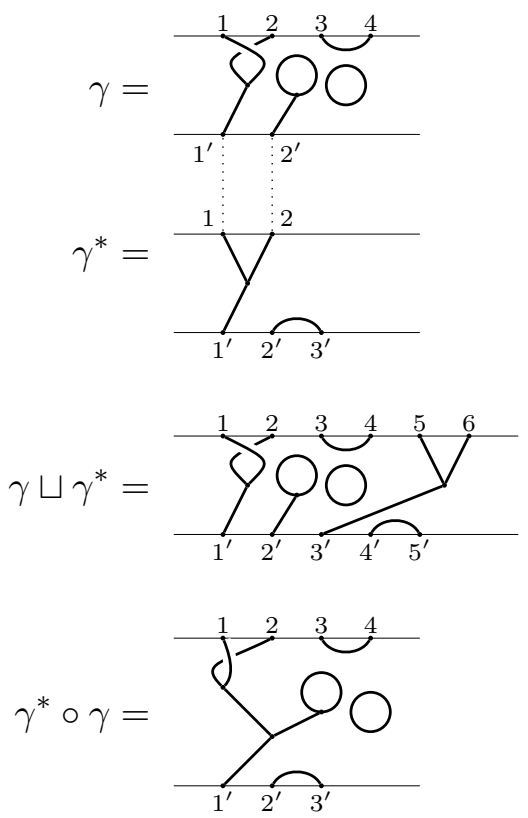

The transpose of a 3 -tangle is obtained rotating it $180^{\circ}$. In CKR05, the transpose is gotten by using a reflection, however, this changes the orientation of the nodes of valency 3 , and we want to avoid that. For $\gamma$ in (3.2), 
its transpose is

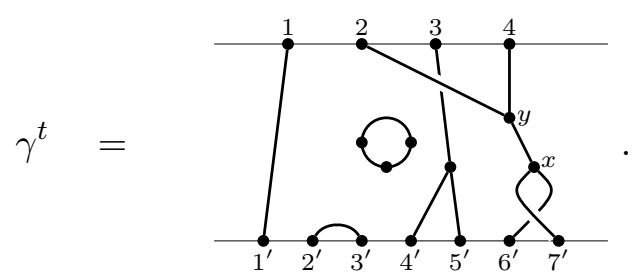

Alternatively, the transpose of $\gamma$ can be obtained as in (2.8) by disjoint union and composition:

$$
\gamma^{t}=\left(\mathrm{I}_{n} \sqcup \beta_{m}\right) \circ\left(\mathrm{I}_{n} \sqcup \gamma \sqcup \mathrm{I}_{m}\right) \circ\left(\beta_{n}^{t} \sqcup \mathrm{I}_{m}\right),
$$

where

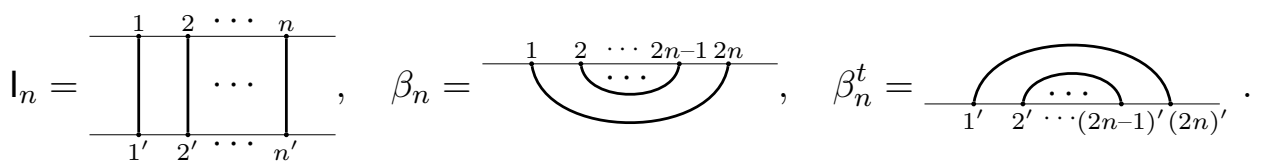

3.2. The 3-tangle categories $\mathcal{T}$ and $\mathcal{T}_{\Gamma}$. Assume $\mathbb{F}$ is a field. The 3tangle category $\mathcal{T}$ has as objects the finite sets $[n], n \in \mathbb{N}$, and as morphisms $\operatorname{Mor}_{\mathcal{T}}([n],[m])$, the $\mathbb{F}$-linear combinations of 3-tangles. The disjoint union $\sqcup$ induces a tensor product $\sqcup: \mathcal{T} \times \mathcal{T} \rightarrow \mathcal{T}$. The tensor product is associative and has the unit object given by the empty 3 -tangle $[0] \rightarrow[0]$.

The transpose induces a map $\operatorname{Mor}_{\mathcal{T}}([n],[m]) \rightarrow \operatorname{Mor}_{\mathcal{T}}([m],[n]), \gamma \mapsto \gamma^{t}$, such that $\left(\gamma^{*} \circ \gamma\right)^{t}=\gamma^{t} \circ\left(\gamma^{*}\right)^{t}$ whenever this makes sense.

Also, as in Proposition 2.5 (see equations (2.6) and (2.7)), there is a bijection

$$
\begin{aligned}
\Phi_{n, m}: \operatorname{Mor}_{\mathcal{T}}([n],[m]) & \longrightarrow \operatorname{Mor}_{\mathcal{T}}([n+m],[0]) \\
\gamma & \mapsto \beta_{m} \circ\left(\gamma \sqcup \mathrm{I}_{n}\right),
\end{aligned}
$$

with inverse

$$
\begin{aligned}
\Psi_{n, m}: \operatorname{Mor}_{\mathcal{T}}([n+m],[0]) & \longrightarrow \operatorname{Mor}_{\mathcal{T}}([n],[m]) \\
\gamma^{\prime} & \mapsto\left(\gamma^{\prime} \sqcup \mathrm{I}_{m}\right) \circ\left(\mathrm{I}_{n} \sqcup \beta_{m}^{t}\right) .
\end{aligned}
$$

The morphisms

$$
\begin{aligned}
& \mathrm{I}_{1}=\begin{array}{l}
\square \\
\end{array} \\
& \beta=\beta_{1}=\smile \\
& \mu=Y \\
& \tau=\lambda \\
& \beta^{t}= \\
& \mu^{t}=\bar{\lambda}
\end{aligned}
$$

are called basic. They constitute the alphabet of $\mathcal{T}$. Note that $\mu^{t}$ can be obtained from the other basic morphisms by composition and tensor product as in (3.3). Also, composing $\mu \circ \tau$, we get

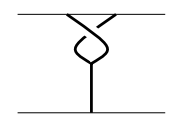

which equals

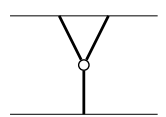


The morphisms in $\mathcal{T}$ are generated, through composition and disjoint union and taking linear combinations, from basic morphisms [CKR05, Proposition 2.3].

In addition to generators, some relations can be imposed in the category $\mathcal{T}$. Let

$$
\Gamma=\left\{\gamma_{i} \in \operatorname{Mor}_{\mathcal{T}}\left(\left[n_{i}\right],\left[m_{i}\right]\right): i=1, \ldots, k\right\}
$$

be a finite set of morphisms in $\mathcal{T}$. For each $n, m \in \mathbb{N}$, the set $\Gamma$ generates, through compositions and tensor products with arbitrary 3-tangles, a subspace $\mathrm{R}_{\Gamma}([n],[m])$ of $\operatorname{Mor}_{\mathcal{T}}([n],[m])$, and we define a new category $\mathcal{T}_{\Gamma}$ with the same objects and with $\operatorname{Mor}_{\mathcal{T}_{\Gamma}}([n],[m])=\operatorname{Mor}_{\mathcal{T}}([n],[m]) / \mathbf{R}_{\Gamma}([n],[m])$. $\mathcal{T}_{\Gamma}$ is the 3-tangle category associated with the set of relations $\Gamma$.

3.3. The functor $\mathcal{R}_{\mathfrak{V}}$. Assume that $\mathfrak{V}=(\mathrm{V}, \mathrm{b}, \mathrm{m})$ is a finite-dimensional nonassociative (i.e. not necessarily associative) algebra with multiplication $\mathrm{m}: \mathrm{V} \times \mathrm{V} \rightarrow \mathrm{V}$ (i.e., $\mathrm{m}$ is an $\mathbb{F}$-bilinear map), and endowed with an associative, nondegenerate, symmetric bilinear form $\mathrm{b}: \mathrm{V} \times \mathrm{V} \rightarrow \mathbb{F}$. Associativity of the form means $\mathrm{b}(\mathrm{m}(x, y), z)=\mathrm{b}(x, \mathrm{~m}(y, z))$ for any $x, y, z \in \mathrm{V}$. Let $\mathcal{V}$ be the category of finite-dimensional vector spaces over $\mathbb{F}$ with linear maps as morphisms. Denote by $\tau$ the switch map $\tau: \mathrm{V}^{\otimes 2} \rightarrow \mathrm{V}^{\otimes 2}, x \otimes y \mapsto y \otimes x$. Recall that we may identify $\mathrm{b}$ with a linear map $\mathrm{V}^{\otimes 2} \rightarrow \mathbb{F}$ and $\mathrm{m}$ with a linear map $\mathrm{V}^{\otimes 2} \rightarrow \mathrm{V}$. Let $1_{\mathrm{V}}$ be the identity map on $\mathrm{V}$.

Theorem 3.8 ([CKR05, Theorem 3.1], [Boo98]). There exists a unique functor $\mathcal{R}_{\mathfrak{V}}: \mathcal{T} \rightarrow \mathcal{V}$ such that:

(1) $\mathcal{R}_{\mathfrak{V}}([0])=\mathbb{F}$ and $\mathcal{R}_{\mathfrak{V}}([n])=\mathrm{V}^{\otimes n}$, for any $n \geq 1$.

(2) $\mathcal{R}_{\mathfrak{V}}\left(\mathrm{I}_{1}\right)=1_{\mathrm{V}}$ and $\mathcal{R}_{\mathfrak{V}}(\tau)=\tau$.

(3) $\mathcal{R}_{\mathfrak{V}}(\beta)=\mathrm{b}, \mathcal{R}_{\mathfrak{V}}(\mu)=\mathrm{m}, \mathcal{R}_{\mathfrak{V}}\left(\gamma^{t}\right)=\mathcal{R}_{\mathfrak{V}}(\gamma)^{t}$, and

$\mathcal{R}_{\mathfrak{V}}(\gamma \sqcup \delta)=\mathcal{R}_{\mathfrak{V}}(\gamma) \otimes \mathcal{R}_{\mathfrak{V}}(\delta)$, for morphisms $\gamma$ and $\delta$ in $\mathcal{T}$.

The symmetry and associativity of the bilinear form b play a key role here.

The empty 3 -tangle $[0] \rightarrow[0]$ corresponds under $\mathcal{R}_{\mathfrak{V}}$ to the identity map in $\operatorname{Hom}_{\mathbb{F}}(\mathbb{F}, \mathbb{F}) \cong \mathbb{F}$. We will denote it simply by 1 .

Remark 3.9. By Remark 2.9,

$$
\mathcal{R}_{\mathfrak{V}}\left(\beta \circ \beta^{t}\right)=\operatorname{dim}_{\mathbb{F}} \vee \in \mathbb{F} \cong \operatorname{Hom}_{\mathbb{F}}(\mathbb{F}, \mathbb{F}) .
$$

For any triple $\mathfrak{V}=(\mathrm{V}, \mathrm{b}, \mathrm{m})$ as above, we have the alphabet of basic tensors $1_{\mathrm{V}}=\mathrm{id}: \mathrm{V} \rightarrow \mathrm{V}, \mathrm{b}: \mathrm{V}^{\otimes 2} \rightarrow \mathbb{F}, \mathrm{m}: \mathrm{V}^{\otimes 2} \rightarrow \mathrm{V}, \tau: \mathrm{V}^{\otimes 2} \rightarrow \mathrm{V}^{\otimes 2}$, $\mathrm{b}^{t}: \mathbb{F} \rightarrow \mathrm{V}^{\otimes 2}$, and $\mathrm{m}^{t}: \mathrm{V} \rightarrow \mathrm{V}^{\otimes 2}$. Let

$$
\Gamma_{\mathfrak{V}}=\left\{\mathrm{c}_{i} \in \operatorname{Hom}_{\mathbb{H}}\left(\mathrm{V}^{\otimes n_{i}}, \mathrm{~V}^{\otimes m_{i}}\right): i=1, \ldots, k\right\}
$$

be a finite set of homomorphisms. The algebra $\mathfrak{V}$ is said to be of tensor type $\Gamma_{\mathfrak{V}}$ if the $c_{i}$ 's are identities for $\mathfrak{V}$, i.e., if

$$
\mathrm{c}_{i}\left(x_{1} \otimes \cdots \otimes x_{n_{i}}\right)=0
$$

for all $i=1, \ldots, k$, and all $x_{1}, \ldots, x_{n_{i}} \in \mathrm{V}$.

Corollary 3.10 ([CKR05, Corollary 3.5]). Let $\mathfrak{V}=(\mathrm{V}, \mathrm{b}, \mathrm{m})$ be an algebra of tensor type $\Gamma_{\mathfrak{V}}=\left\{c_{i}: i=1, \ldots, k\right\}$, for tensors $c_{i}$ expressible in terms of the alphabet, $1_{\mathrm{V}}, \mathrm{m}, \mathrm{m}^{t}, \mathrm{~b}, \mathrm{~b}^{t}$, and $\tau$. Let $\Gamma=\left\{\gamma_{i}: i=1, \ldots, k\right\}$ with 
$\mathcal{R}_{\mathfrak{V}}\left(\gamma_{i}\right)=\mathrm{c}_{i}$ for all $i=1, \ldots, k$. Then there is a unique functor $\mathcal{R}_{\Gamma}: \mathcal{T}_{\Gamma} \rightarrow \mathcal{V}$ such that $\mathcal{R}_{\mathcal{V}}=\mathcal{R}_{\Gamma} \circ \mathcal{P}$, where $\mathcal{P}$ is the natural projection $\mathcal{T} \rightarrow \mathcal{T}_{\Gamma}$.

\section{SEVEn-Dimensional CROSS PRODUCtS}

4.1. Cross products. Let $\mathbb{F}$ be a field of characteristic $\neq 2$, and let $(\mathrm{V}, \mathrm{b})$ be a vector space $\mathrm{V}$ over $\mathbb{F}$ equipped with a nondegenerate symmetric bilinear form $\mathrm{b}$. A cross product on $(\mathrm{V}, \mathrm{b})$ is a bilinear multiplication $\mathrm{V} \times \mathrm{V} \rightarrow \mathrm{V}$, $(x, y) \mapsto x \times y$, such that:

$$
\begin{aligned}
& \mathrm{b}(x \times y, x)=0, \\
& x \times x=0, \\
& \mathrm{~b}(x \times y, x \times y)=\mathrm{b}(x, x) \mathrm{b}(y, y)-\mathrm{b}(x, y) \mathrm{b}(y, x),
\end{aligned}
$$

for any $x, y \in \mathrm{V}$. It is well known that a nonzero cross product exists only if $\operatorname{dim}_{\mathbb{F}} \mathrm{V}=3$ or 7 . (See [BG67]. For a proof using 3-tangles, see [Ros04].)

Since the characteristic is $\neq 2$, the equations in (4.1) are equivalent to:

$$
\begin{aligned}
& \mathrm{b}(x \times y, z)=\mathrm{b}(x, y \times z), \\
& x \times y=-y \times x, \\
& \mathrm{~b}(x \times y, z \times t)+\mathrm{b}(y \times z, t \times x)= \\
& \quad 2 \mathrm{~b}(x, z) \mathrm{b}(y, t)-\mathrm{b}(x, y) \mathrm{b}(z, t)-\mathrm{b}(y, z) \mathrm{b}(x, t),
\end{aligned}
$$

for any $x, y, z, t \in \mathrm{V}$. Equation (4.2a) shows that $\mathrm{b}$ is associative relative to the cross product, so that $\mathfrak{V}=(\mathrm{V}, \mathrm{b}, \times)$ satisfies the conditions in Section 3. and (4.2b) and (4.2c) can be expressed as

$$
\mathrm{c}_{1}\left(x_{1} \otimes x_{2}\right)=0=\mathrm{c}_{2}\left(x_{1} \otimes x_{2} \otimes x_{3} \otimes x_{4}\right)
$$

for any $x_{1}, x_{2}, x_{3}, x_{4} \in \mathrm{V}$, where

$$
\begin{aligned}
& \mathrm{c}_{1}\left(x_{1} \otimes x_{2}\right)=x_{1} \times x_{2}+x_{2} \times x_{1}, \\
& \mathrm{c}_{2}\left(x_{1} \otimes x_{2} \otimes x_{3} \otimes x_{4}\right)=\mathrm{b}\left(x_{1} \times x_{2}, x_{3} \times x_{4}\right)+\mathrm{b}\left(x_{2} \times x_{3}, x_{4} \times x_{1}\right) \\
& \quad-2 \mathrm{~b}\left(x_{1}, x_{3}\right) \mathrm{b}\left(x_{2}, x_{4}\right)+\mathrm{b}\left(x_{1}, x_{2}\right) \mathrm{b}\left(x_{3}, x_{4}\right)+\mathrm{b}\left(x_{2}, x_{3}\right) \mathrm{b}\left(x_{1}, x_{4}\right) .
\end{aligned}
$$

Thus, $c_{1} \in \operatorname{Hom}_{\mathbb{F}}\left(\mathrm{V}^{\otimes 2}, \mathrm{~V}\right)$ and $\mathrm{c}_{2} \in \mathrm{Hom}_{\mathbb{F}}\left(\mathrm{V}^{\otimes 4}, \mathbb{F}\right)$, and they are the images under $\mathcal{R}_{\mathfrak{V}}$ of $\gamma_{1} \in \operatorname{Mor}_{\mathcal{T}}([2],[1])$ and $\gamma_{2}^{\prime} \in \operatorname{Mor}_{\mathcal{T}_{2}}([4],[0])$, with

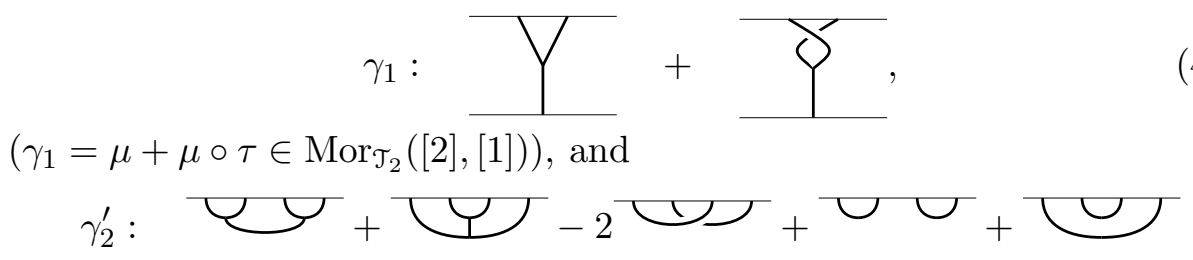

Using $\Psi_{2,2}$ we may substitute $\gamma_{2}^{\prime}$ with

$\gamma_{2}:$

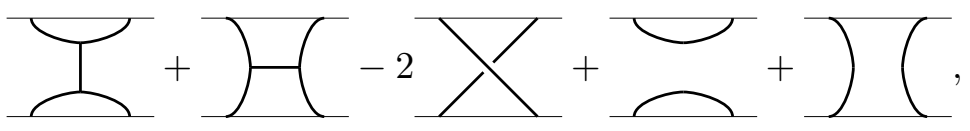

where $\gamma_{2} \in \operatorname{Mor}_{\mathcal{T}}([2],[2])$.

Also, Remarks 2.9 and 3.9 show that $\left(\mathrm{b} \circ \mathrm{b}^{t}\right)(1)=\operatorname{dim}_{\mathbb{F}} \mathrm{V} \neq 0$ and

$$
\mathrm{c}_{0}=\left(\mathrm{b} \circ \mathrm{b}^{t}\right)(1)-\operatorname{dim}_{\mathbb{F}} \mathrm{V} \in \mathbb{F} \simeq \operatorname{Hom}_{\mathbb{F}}(\mathbb{F}, \mathbb{F})
$$


is the image under $\mathcal{R}_{\mathfrak{V}}$ of

$$
\gamma_{0}=\beta \circ \beta^{t}-\left(\operatorname{dim}_{\mathbb{F}} \mathrm{V}\right) 1=\bigcirc-\left(\operatorname{dim}_{\mathbb{F}} \mathrm{V}\right) 1, \quad \gamma_{0} \in \operatorname{Mor}_{\mathcal{T}}([0],[0])
$$

Therefore, we have the following consequence of Corollary 3.10 .

Proposition 4.8. If $\mathfrak{V}=(\mathrm{V}, \mathrm{b}, \times)$ for a vector space $\mathrm{V}$ endowed with a nonzero cross product $\times$ relative to the nondegenerate symmetric bilinear form $\mathrm{b}$, then $\mathfrak{V}$ is of tensor type $\left\{\mathrm{c}_{0}, \mathrm{c}_{1}, \mathrm{c}_{2}\right\}$, for $\mathrm{c}_{0}$ in (4.6) and $\mathrm{c}_{1}$ and $\mathrm{c}_{2}$ in (4.3), and hence the functor $\mathcal{R}_{\mathfrak{V}}$ induces a functor $\mathcal{R}_{\Gamma}: \mathcal{T}_{\Gamma} \rightarrow \mathcal{V}$, with $\Gamma=\left\{\gamma_{0}, \gamma_{1}, \gamma_{2}\right\}$, for $\gamma_{0}$ in (4.7), $\gamma_{1}$ in (4.4), and $\gamma_{2}$ in (4.5).

Equation (4.1c) is equivalent, by the associativity and nondegeneracy of $\mathrm{b}$ and the anticommutativity of $\times$, to the equation:

$$
(x \times y) \times z+x \times(y \times z)=2 \mathrm{~b}(x, z) y-\mathrm{b}(x, y) z-\mathrm{b}(y, z) x,
$$

for any $x, y, z \in \mathrm{V}$. From here it is easy to check that any automorphism of $(\mathrm{V}, \times)$ is an isometry of $(\mathrm{V}, \mathrm{b})$. Then $\mathrm{b}, \mathrm{b}^{t}, \tau$ and $\times$ are invariant under the action of Aut $(V, \times)$, which is a simple algebraic group of type $G_{2}$ if $\operatorname{dim}_{\mathbb{F}} \mathrm{V}=7$ and is the special orthogonal group $\mathrm{SO}(\mathrm{V}, \mathrm{b})$ if $\operatorname{dim}_{\mathbb{F}} \mathrm{V}=3$.

4.2. Dimension 7 cross products. Suppose now that $\operatorname{dim}_{\mathbb{F}} \mathrm{V}=7$. Then the image $\mathcal{R}_{\Gamma}\left(\operatorname{Mor}_{\mathcal{T}_{\Gamma}}([n],[m])\right)$, which lies in $\operatorname{Hom}_{\mathbb{F}}\left(\mathrm{V}^{\otimes n}, \mathrm{~V}^{\otimes m}\right)$, is actually contained in $\operatorname{Hom}_{\text {Aut }(\mathrm{V}, \times)}\left(\mathrm{V}^{\otimes n}, \mathrm{~V}^{\otimes m}\right)$, the subspace of linear maps invariant under the action of Aut $(\mathrm{V}, \times)$.

Theorem 4.10. Let $\mathbb{F}$ be an infinite field of characteristic $\neq 2,3$, and let $\mathfrak{V}=(\mathrm{V}, \mathrm{b}, \times)$ be a 7 -dimensional vector space $\mathrm{V}$ endowed with a nonzero cross product $x \times y$ relative to the nondegenerate symmetric bilinear form $\mathrm{b}$. For any $n, m \in \mathbb{N}$,

$$
\mathcal{R}_{\Gamma}\left(\operatorname{Mor}_{\mathcal{T}_{\Gamma}}([n],[m])\right)=\operatorname{Hom}_{\text {Aut }(\mathrm{V}, \times)}\left(\mathrm{V}^{\otimes n}, \mathrm{~V}^{\otimes m}\right) .
$$

Proof. The algebra $\mathrm{C}=\mathbb{F} 1 \oplus \mathrm{V}$, with multiplication

$$
(\alpha 1+v)(\beta 1+w)=(\alpha \beta-\mathbf{b}(v, w)) 1+(\alpha w+\beta v+v \times w)
$$

for $\alpha, \beta \in \mathbb{F}$ and $v, w \in \mathrm{V}$, is a Cayley algebra over $\mathbb{F}$. Any $x \in \mathrm{C}$ satisfies the Cayley-Hamilton equation:

$$
x^{2}-\operatorname{tr}(x) x+\mathrm{n}(x) 1=0,
$$

where the trace and norm are given by

$$
\operatorname{tr}(\alpha 1+v)=2 \alpha, \quad \mathrm{n}(\alpha 1+v)=\alpha^{2}+\mathrm{b}(v, v) .
$$

(See [Scha66, Chapter III] for basic facts on Cayley algebras.)

Denote by $\mathbb{F}[n \mathrm{~V}]$ the algebra of polynomial maps on the Cartesian power $\mathrm{V}^{n}$. According to [Schw88, (3.23)] and [ZSh15], the First Fundamental Theorem of Invariant Theory for $G_{2}$ asserts that the algebra of invariants $\mathbb{F}[n \mathrm{~V}]^{\text {Aut }(\mathrm{V}, \times)}$ is generated by the polynomial maps

$$
\begin{aligned}
& \left(v_{1}, \ldots, v_{n}\right) \stackrel{\alpha_{i j}}{\longrightarrow} \operatorname{tr}\left(v_{i} v_{j}\right), \\
& \left(v_{1}, \ldots, v_{n}\right) \stackrel{\beta_{i j k}}{\longrightarrow} \operatorname{tr}\left(v_{i}\left(v_{j} v_{k}\right)\right), \\
& \left(v_{1}, \ldots, v_{n}\right) \stackrel{\gamma_{i j k l}}{\longrightarrow} \text { skew } \operatorname{tr}\left(v_{i}\left(v_{j}\left(v_{k} v_{l}\right)\right)\right) .
\end{aligned}
$$


Here skew $f\left(x_{1}, x_{2}, x_{3}, x_{4}\right)=\sum_{\sigma \in \mathrm{S}_{4}}(-1)^{\sigma} f\left(x_{\sigma(1)}, x_{\sigma(2)}, x_{\sigma(3)}, x_{\sigma(4)}\right)$, denotes the complete skew symmetrization of the map $f$ relative to the symmetric group $\mathrm{S}_{4}$, and $(-1)^{\sigma}$ is the sign of the permutation $\sigma$.

Let us note that for any $n \in \mathbb{N}, n \geq 2$, and any $v_{1}, \ldots, v_{n} \in \mathrm{V}$,

$$
\alpha_{i j}\left(v_{1}, \ldots, v_{n}\right)=-2 \mathrm{~b}\left(v_{i}, v_{j}\right) \text {. }
$$

Also,

$$
\begin{aligned}
v_{i}\left(v_{j} v_{k}\right) & =-\mathrm{b}\left(v_{j}, v_{k}\right) v_{i}+v_{i}\left(v_{j} \times v_{k}\right) \\
& =-\mathrm{b}\left(v_{j}, v_{k}\right) v_{i}-\mathrm{b}\left(v_{i}, v_{j} \times v_{k}\right) 1+v_{i} \times\left(v_{j} \times v_{k}\right),
\end{aligned}
$$

and since $\operatorname{tr}(v)=0$ for any $v \in \mathrm{V}$, it follows that for $n \geq 3$,

$$
\beta_{i j k}\left(v_{1}, \ldots, v_{n}\right)=-\mathrm{b}\left(v_{i}, v_{j} \times v_{k}\right) .
$$

Finally,

$$
\begin{aligned}
\operatorname{tr}\left(v_{i}\left(v_{j}\left(v_{k} v_{l}\right)\right)\right) & =\operatorname{tr}\left(\left(v_{i} v_{j}\right)\left(v_{k} v_{l}\right)\right) \\
& =\operatorname{tr}\left(\left(-\mathrm{b}\left(v_{i}, v_{j}\right) 1+v_{i} \times v_{j}\right)\left(-\mathrm{b}\left(v_{k}, v_{l}\right) 1+v_{k} \times v_{l}\right)\right) \\
& =2 \mathrm{~b}\left(v_{i}, v_{j}\right) \mathrm{b}\left(v_{k}, v_{l}\right)-2 \mathrm{~b}\left(v_{i} \times v_{j}, v_{k} \times v_{l}\right) .
\end{aligned}
$$

The skew symmetrization of the first summand is 0 , and because $b$ is symmetric and $\times$ is anticommutative, the skew symmetrization of $\mathrm{b}\left(v_{i} \times v_{j}, v_{k} \times v_{l}\right)$ is 8 times

$$
\begin{aligned}
\mathrm{b}\left(v_{i} \times v_{j}, v_{k} \times v_{l}\right) & +\mathrm{b}\left(v_{j} \times v_{k}, v_{i} \times v_{l}\right)+\mathrm{b}\left(v_{k} \times v_{i}, v_{j} \times v_{l}\right) \\
= & \mathrm{b}\left(\left(v_{i} \times v_{j}\right) \times v_{l}+\left(v_{j} \times v_{k}\right) \times v_{i}+\left(v_{k} \times v_{i}\right) \times v_{j}, v_{l}\right) .
\end{aligned}
$$

But because of (4.1), we get:

$$
\begin{aligned}
\left(v_{j} \times v_{k}\right) \times v_{i} & =-\left(v_{k} \times v_{j}\right) \times v_{i} \\
& =v_{k} \times\left(v_{j} \times v_{i}\right)-2 \mathrm{~b}\left(v_{i}, v_{k}\right) v_{j}+\mathrm{b}\left(v_{j}, v_{i}\right) v_{k}+\mathrm{b}\left(v_{j}, v_{k}\right) v_{i} \\
& =\left(v_{i} \times v_{j}\right) \times v_{k}+\mathrm{b}\left(v_{i}, v_{j}\right) v_{k}-2 \mathrm{~b}\left(v_{i}, v_{k}\right) v_{j}+\mathrm{b}\left(v_{j}, v_{k}\right) v_{i}, \\
\left(v_{k} \times v_{i}\right) \times v_{j} & =-v_{k} \times\left(v_{i} \times v_{j}\right)+2 \mathrm{~b}\left(v_{k}, v_{j}\right) v_{i}-\mathrm{b}\left(v_{k}, v_{i}\right) v_{j}-\mathrm{b}\left(v_{i}, v_{j}\right) v_{k} \\
& =\left(v_{i} \times v_{j}\right) \times v_{k}-\mathrm{b}\left(v_{i}, v_{j}\right) v_{k}-\mathrm{b}\left(v_{i}, v_{k}\right) v_{j}+2 \mathrm{~b}\left(v_{j}, v_{k}\right) v_{i} .
\end{aligned}
$$

Hence, the complete skew symmetrization of $\mathrm{b}\left(v_{i} \times v_{j}, v_{k} \times v_{l}\right)$ is 24 times

$$
\mathrm{b}\left(\left(v_{i} \times v_{j}\right) \times v_{k}, v_{l}\right)-\mathrm{b}\left(v_{i}, v_{k}\right) \mathrm{b}\left(v_{j}, v_{l}\right)+\mathrm{b}\left(v_{i}, v_{l}\right) \mathrm{b}\left(v_{j}, v_{k}\right) .
$$

Therefore, the above quoted results of Schwarz and Zubkov-Shestakov show that the algebra of invariants $\mathbb{F}[n \mathrm{~V}]^{\text {Aut }(\mathrm{V}, \times)}$ is generated by the polynomial maps:

$$
\begin{aligned}
& \left(v_{1}, \ldots, v_{n}\right) \longrightarrow \mathrm{b}\left(v_{i}, v_{j}\right), \\
& \left(v_{1}, \ldots, v_{n}\right) \longrightarrow \mathrm{b}\left(v_{i}, v_{j} \times v_{k}\right), \\
& \left(v_{1}, \ldots, v_{n}\right) \longrightarrow \mathrm{b}\left(v_{i} \times v_{j}, v_{k} \times v_{l}\right),
\end{aligned}
$$

and we conclude that the space $\operatorname{Hom}_{\mathrm{Aut}(\mathrm{V}, \times)}\left(\mathrm{V}^{\otimes n}, \mathrm{~V}^{\otimes m}\right)$, which is isomorphic to $\operatorname{Hom}_{\text {Aut }(\mathrm{V}, \times)}\left(\mathrm{V}^{\otimes(n+m)}, \mathbb{F}\right)$, i.e., the space of multilinear maps $\mathrm{V}^{n+m} \rightarrow \mathbb{F}$ invariant under the action of $\operatorname{Aut}(\mathrm{V}, \times)$, is the linear span of the set of linear maps $\mathrm{V}^{\otimes n} \rightarrow \mathrm{V}^{\otimes m}$ generated by composition and tensor products by $1_{\mathrm{V}}, \mathrm{b}$, $\mathrm{b}^{t}, \tau$ and $\times$. This is precisely $\mathcal{R}_{\Gamma}\left(\operatorname{Mor}_{\mathcal{T}_{\Gamma}}([n],[m])\right)$. 
Our goal now is to prove that $\mathcal{R}_{\Gamma}$ actually gives a bijection $\operatorname{Mor}_{\mathcal{T}_{\Gamma}}([n],[m]) \rightarrow$ $\operatorname{Hom}_{\text {Aut }(\mathrm{V}, \times)}\left(\mathrm{V}^{\otimes n}, \mathrm{~V}^{\otimes m}\right)$ and to find bases of these spaces. We will start by working in $\mathcal{T}_{\Gamma}$.

The morphisms of $\mathcal{T}_{\Gamma}$ are the equivalence classes of 3-tangles modulo the relations in $\Gamma=\left\{\gamma_{0}, \gamma_{1}, \gamma_{2}\right\}$, so we will attempt to get a set of 3 -tangles $\gamma:[n] \rightarrow[m]$ that are representatives of a spanning set of $\operatorname{Mor}_{\mathcal{T}_{\Gamma}}([n],[m])$. Later on, this spanning set will be shown to be a basis.

We will use the same symbols to denote morphisms in $\mathcal{T}$ and their images in $\mathcal{T}_{\Gamma}$. Our argument will involve using the following steps aimed at

eliminating circles, crossings, triangles, squares and pentagons.

1. Since $\gamma_{0}$ is one of the relations, we can get rid of circles, i.e., any 3tangle modulo $\Gamma$ equals a 3 -tangle without circles. Also, the relation $\gamma_{2}$ allows us to suppress any crossings $\searrow$, i.e., a 3-tangle with crossings is equivalent modulo $\Gamma$ to a linear span of 3 -tangles without crossings.

Note that if there are no crossings, a 3 -tangle is the disjoint union of its connected components:

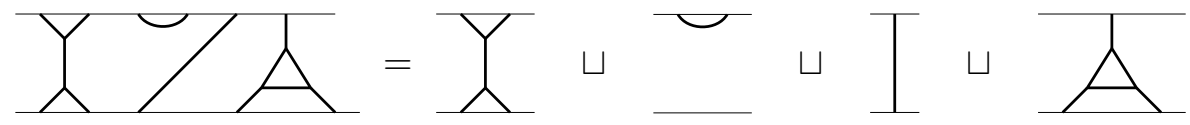

2. In $\mathcal{T}_{\Gamma}$ we have:

$$
\begin{gathered}
\mu \circ \tau \circ \beta^{t}= \begin{cases}\mu \circ\left(\tau \circ \beta^{t}\right)=\mu \circ \beta^{t} & \text { because } \tau \circ \beta^{t}=\beta^{t}, \\
(\mu \circ \tau) \circ \beta^{t}=-\mu \circ \beta^{t} & \text { because of relation } \gamma_{1} .\end{cases} \\
P=-P
\end{gathered}
$$

Therefore, in $\mathcal{T}_{\Gamma}, \mu \circ \beta^{t}=0$ :

$$
\Upsilon=0
$$

3. Let us prove next (see [Ros04]) that the following relation holds:

$$
\Upsilon_{1}=-6
$$

Indeed, relation $\gamma_{2}$ gives:

$$
\begin{aligned}
& \because=-O+2 O-O+O \\
& =0+2|-\bigcirc|-\mid \\
& =\left(1-\operatorname{dim}_{\mathbb{F}} \mathrm{V}\right)|=-6| \text {. }
\end{aligned}
$$


4. Again with relation $\gamma_{2}$, we get:

$$
\begin{aligned}
Y+Y & =2 Y-Y-\bar{Q} \\
& =-3 Y \quad \text { because of } \gamma_{1} \text { and Step } 2,
\end{aligned}
$$

and because of Step 3, we conclude

$$
Y=3 Y
$$

5. A new application of relation $\gamma_{2}$ gives

$$
\begin{aligned}
& \underline{\mu}=-\bar{\lambda}+2 \zeta-\rangle-\overline{\mathrm{O}}
\end{aligned}
$$

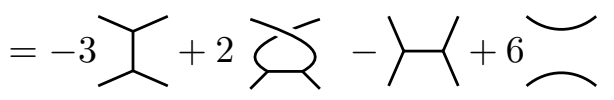

and

$$
\begin{aligned}
& \vartheta=-\Phi+2 \Phi-\lambda-\underline{O}
\end{aligned}
$$

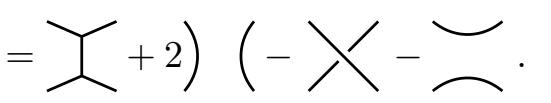

Thus, we have

$$
\underline{Z}=-\boldsymbol{Y}-\rangle(+4)(-2)
$$

and applying relation $\gamma_{2}$ again gives

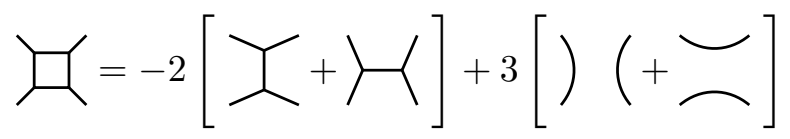

6. Finally, relation $\gamma_{2}$, together with the previous steps, shows:

$$
\text { से=- }
$$


and

$$
\begin{aligned}
y \\
y
\end{aligned}
$$

Hence,

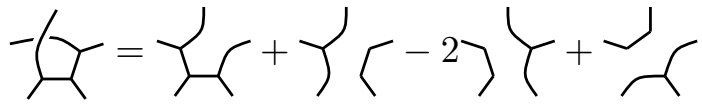

$$
\begin{aligned}
& +2 \lambda-\frac{1}{1}+\gamma y
\end{aligned}
$$

and consequently,

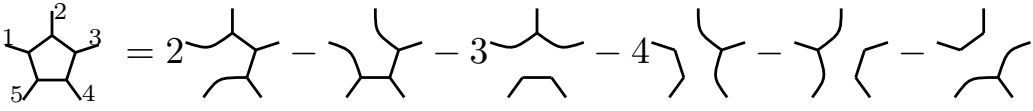

$$
\begin{aligned}
& +2 \gamma y-2 \frac{1}{1}+4 \lambda \\
& =2 a_{3}-a_{4}-3 b_{2}-4 b_{3}-b_{1}-b_{4}+2 c_{1}-2 c_{2}+4 c_{5} \text {, }
\end{aligned}
$$

where

$$
a_{1}=\underbrace{1}, \quad b_{1}=Y^{\prime}, \quad c_{1}=1) Y,
$$

and the rest are cyclic permutations of them. But from relation $\gamma_{2}$, we obtain

$$
2 y=Y(+Y)+r(+) Y
$$

that is:

$$
2 c_{1}=a_{5}+a_{2}+b_{4}+b_{3} .
$$

Permuting cyclically, we get:

$$
\begin{aligned}
2 c_{1} & =a_{2}+a_{5}+b_{3}+b_{4}, \\
-2 c_{2} & =-a_{3}-a_{1}-b_{4}-b_{5}, \\
4 c_{5} & =2 a_{1}+2 a_{4}+2 b_{2}+2 b_{3},
\end{aligned}
$$

and, therefore,

$$
\begin{gathered}
\mathcal{Y}=2 a_{3}-a_{4}-3 b_{2}-4 b_{3}-b_{1}-b_{4}+2 c_{1}-2 c_{2}+4 c_{5} \\
=\left[a_{1}+a_{2}+a_{3}+a_{4}+a_{5}\right]-\left[b_{1}+b_{2}+b_{3}+b_{4}+b_{5}\right],
\end{gathered}
$$


that is,

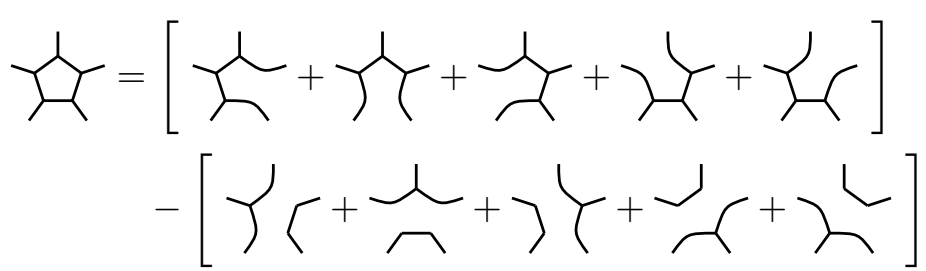

The reader may compare the relations obtained so far with the results in [Kup94, Theorem 1.1] and Kup96 for $q=1$.

Remark 4.12. The 3 -tangles $[n] \rightarrow[0]$ without subgraphs of the form

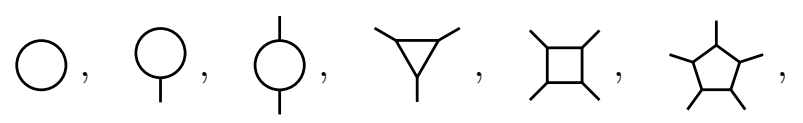

correspond bijectively with the nonpositive diagrams of Wes07, Definition 4.2]. Steps 1-6 show that such 3-tangles form a spanning set of $\operatorname{Mor}_{\mathcal{T}_{\Gamma}}([n],[0])$.

Theorem 4.14. Let $n, m \in \mathbb{N}$, and assume that the characteristic of $\mathbb{F}$ is 0 . Let $\Gamma=\left\{\gamma_{0}, \gamma_{1}, \gamma_{2}\right\}$, where $\gamma_{0}$ is as in (4.7), $\gamma_{1}$ as in (4.4), and $\gamma_{2}$ as in (4.5).

(a) The classes modulo $\Gamma$ of the 3 -tangles $[n] \rightarrow[m]$ without crossings and without any of the subgraphs in (4.13) form a basis of $\operatorname{Mor}_{\mathcal{T}_{\Gamma}}([n],[m])$.

(b) The functor $\mathcal{R}_{\Gamma}$ from Corollary 3.10 gives a linear isomorphism

$$
\operatorname{Mor}_{\mathcal{T}_{\Gamma}}([n],[m]) \rightarrow \operatorname{Hom}_{\text {Aut }(\mathrm{V}, \times)}\left(\mathrm{V}^{\otimes n}, \mathrm{~V}^{\otimes m}\right),
$$

where $\mathfrak{V}=(\mathrm{V}, \mathrm{b}, \times)$ is a 7 -dimensional vector space $\mathrm{V}$ endowed with a nonzero cross product $x \times y$ relative to the nondegenerate symmetric bilinear form $\mathrm{b}$, and $\mathrm{Aut}(\mathrm{V}, \times)$ is a simple algebraic group of type $\mathrm{G}_{2}$.

(c) The 3-tangles $[n] \rightarrow[n]$ as in part (a) give a basis of the centralizer algebra

$$
\operatorname{End}_{\text {Aut }(\mathrm{V}, \times)}\left(\mathrm{V}^{\otimes n}\right) \simeq \operatorname{Mor}_{\mathcal{T}_{\Gamma}}([n],[n]) .
$$

Proof. The results in Kup96, Theorems 5.1 and 6.10] or [Wes07] (which are valid in characteristic 0 ) show that for any $n \in \mathbb{N}$, the dimension of $\operatorname{Hom}_{\text {Aut }(\mathrm{V}, \times)}\left(\mathrm{V}^{\otimes(n+m)}, \mathbb{F}\right)$ coincides with the number of 3-tangles $[n+m] \rightarrow$ $[0]$ without crossings and subgraphs in (4.13). Since $\mathcal{R}_{\Gamma}$ gives a surjective linear isomorphism $\operatorname{Mor}_{\mathcal{T}_{\Gamma}}([n+m],[0]) \rightarrow \operatorname{Hom}_{\text {Aut }(\mathrm{V}, \times)}\left(\mathrm{V}^{\otimes(n+m)}, \mathbb{F}\right)$, this proves that this map is a bijection.

The theorem now follows using the linear isomorphisms

$$
\Phi_{n, m}: \operatorname{Hom}_{\mathbb{F}}\left(\mathrm{V}^{\otimes n}, \mathrm{~V}^{\otimes m}\right) \rightarrow \operatorname{Hom}_{\mathbb{F}}\left(\mathrm{V}^{\otimes(n+m)}, \mathbb{F}\right)
$$

in Proposition 2.5 and the bijection

$$
\Phi_{n, m}: \operatorname{Mor}_{\mathcal{T}_{\Gamma}}([n],[m]) \rightarrow \operatorname{Mor}_{\mathcal{T}_{\Gamma}}([n+m],[0])
$$

induced by the one in (2.6) , noting that this bijection sends 3 -tangles $[n] \rightarrow$ $[m]$ without crossings and any of the subgraphs in (4.13) to 3-tangles $[n+$ $m] \rightarrow[0]$ with the same properties. 
Remark 4.15. The sequence $c(n)=\operatorname{dim}_{\mathbb{F}} \operatorname{Mor}_{\mathcal{T}_{\Gamma}}([n],[0])$ is the sequence \#A059710 in OEIS]. The dimension of $\operatorname{Mor}_{\mathcal{T}_{\Gamma}}([n],[m]) \simeq \operatorname{Mor}_{\mathcal{T}_{\Gamma}}([n+m],[0])$ is then $c(n+m)$.

Remark 4.16. Multiplication in the basis of part (c) of this theorem corresponds to composing by bordism and applying Steps 1-6 of (4.11). One has to suppress circles and cycles of arbitrary length and normalize the resulting 3 -tangles using the arguments prior to Theorem 4.10.

Remark 4.17. Relation $\gamma_{2}$ gives that in $\mathcal{T}_{\Gamma}$,

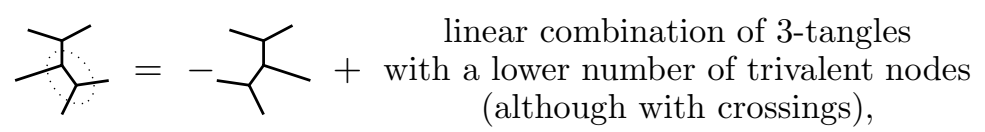

but the graph on the right is obtained by rotating counterclockwise the graph on the left an angle of $\frac{4 \pi}{3}$. If we then repeat the argument five times, we obtain

$$
Y=-Y+\begin{gathered}
\text { linear combination of } 3 \text {-tangles } \\
\text { with a lower number of trivalent nodes, }
\end{gathered}
$$

so we get

$$
Y=\begin{gathered}
\text { linear combination of } 3 \text {-tangles } \\
\text { with a lower number of trivalent nodes. }
\end{gathered}
$$

This proves that $\operatorname{Mor}_{\mathcal{T}_{\Gamma}}([n],[0])$ is spanned by 3 -tangles where crossings are allowed, but where each connected component has at most two trivalent nodes, so each connected component is of the form:

- $\underbrace{j}$, which corresponds under $\mathcal{R}_{\Gamma}$ to $\mathrm{b}\left(v_{i}, v_{j}\right)$,

- $\underbrace{j}$, , which corresponds under $\mathcal{R}_{\Gamma}$ to $\mathrm{b}\left(v_{i} \times v_{j}, v_{k}\right)$, and

- $\underbrace{j} \zeta^{l}$, which corresponds under $\mathcal{R}_{\Gamma}$ to $\mathrm{b}\left(v_{i} \times v_{j}, v_{k} \times v_{l}\right)$.

Hence, from the fact that $\mathcal{R}_{\Gamma}$ gives a linear surjection $\operatorname{Mor}_{\mathcal{T}_{\Gamma}}([n],[0]) \rightarrow$ $\operatorname{Hom}_{\text {Aut }(\mathrm{V}, \times)}\left(\mathrm{V}^{\otimes n}, \mathbb{F}\right)$, which follows from the arguments in the proof of Kup96, Theorem 5.1], we recover the First Fundamental Theorem of Invariant Theory for $\mathrm{G}_{2}$ in characteristic 0 [Schw88], used at the beginning of Theorem 4.10

The 3-tangles with crossings and connected components with at most two trivalent nodes do not form a basis of the spaces $\operatorname{Mor}_{\mathcal{T}_{\Gamma}}([n],[m])$.

\section{ThreE-DIMENSIONAL CROSS PRODUCTS}

In this section, $\mathfrak{V}=(\mathrm{V}, \mathrm{b}, \times)$ will consist of a 3-dimensional vector space over a field $\mathbb{F}$ of characteristic $\neq 2$, endowed with a nonzero cross product $x \times y$, relative to a nondegenerate symmetric bilinear form $\mathrm{b}$.

Then the following conditions hold:

$$
\begin{aligned}
& \mathrm{b}(x \times y, z)=\mathrm{b}(x, y \times z), \\
& x \times y=-y \times x, \\
& (x \times y) \times z=\mathrm{b}(x, z) y-\mathrm{b}(y, z) x,
\end{aligned}
$$


for all $x, y, z \in \mathrm{V}$. Conditions (5.1b) and (5.1c) can be expressed as $\widehat{\mathrm{c}}_{1}\left(x_{1} \otimes x_{2}\right)=0=\widehat{\mathrm{c}}_{2}\left(x_{1} \otimes x_{2} \otimes x_{3}\right)$ for any $x_{1}, x_{2}, x_{3} \in \mathrm{V}$, where

$$
\begin{aligned}
& \widehat{\mathrm{c}}_{1}\left(x_{1} \otimes x_{2}\right)=x_{1} \times x_{2}+x_{2} \times x_{1}, \\
& \widehat{\mathrm{c}}_{2}\left(x_{1} \otimes x_{2} \otimes x_{3}\right)=\left(x_{1} \times x_{2}\right) \times x_{3}-\mathrm{b}\left(x_{1}, x_{3}\right) x_{2}+\mathrm{b}\left(x_{2}, x_{3}\right) x_{1},
\end{aligned}
$$

so $\widehat{c}_{1} \in \operatorname{Hom}_{\mathbb{F}}\left(V^{\otimes 2}, \mathrm{~V}\right)$ and $\widehat{c}_{2} \in \operatorname{Hom}_{\mathbb{F}}\left(V^{\otimes 3}, \mathrm{~V}\right)$, and under $\mathcal{R}_{\mathcal{V}}$, these are the images respectively of

$$
\widehat{\gamma}_{1}=\mu+\mu \circ \tau \in \operatorname{Mor}_{\mathcal{T}_{2}}([2],[1])
$$

and of

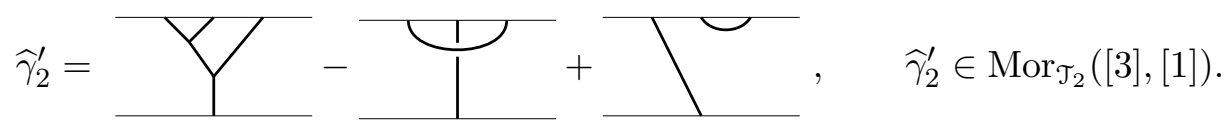

Let us consider, instead of $\widehat{\gamma}_{2}^{\prime}$, the 3 -tangle

$$
\widehat{\gamma}_{2}=\left(\hat{\gamma}_{2}^{\prime} \sqcup \mathrm{I}_{1}\right) \circ\left(\mathrm{I}_{2} \sqcup \beta_{2}^{t}\right): \square+
$$

where $\widehat{\gamma}_{2} \in \operatorname{Mor}_{\mathcal{T}_{2}}([2],[2])$. (Conversely, we can recover $\widehat{\gamma}_{2}^{\prime}$ from $\widehat{\gamma}_{2}$ by composition and disjoint union.)

Also,

$$
\widehat{c}_{0}=\left(b \circ b^{t}\right)(1)-\operatorname{dim}_{\mathbb{F}} V=\left(b \circ b^{t}\right)(1)-3 \in \mathbb{F} \simeq \operatorname{Hom}_{\mathbb{F}}(\mathbb{F}, \mathbb{F})
$$

is the image of

$$
\widehat{\gamma}_{0}:=\beta \circ \beta^{t}-3: \bigcirc-3 \cdot 1, \quad \widehat{\gamma}_{0} \in \operatorname{Mor}_{\mathcal{T}_{2}}([0],[0]) .
$$

Therefore, for $\mathfrak{V}=(\mathrm{V}, \mathrm{b}, \times)$ as above, $\mathfrak{V}$ is of tensor type $\left\{\widehat{\mathrm{c}}_{0}, \widehat{\mathrm{c}}_{1}, \widehat{\mathrm{c}}_{2}\right\}$, and hence Corollary 3.10 gives a functor $\mathcal{R}_{\widehat{\Gamma}}: \mathcal{T}_{\widehat{\Gamma}} \rightarrow \mathcal{V}$, with $\widehat{\Gamma}=\left\{\widehat{\gamma}_{0}, \widehat{\gamma}_{1}, \widehat{\gamma}_{2}\right\}$. As in the previous section, the image $\mathcal{R}_{\widehat{\Gamma}}\left(\operatorname{Mor}_{\mathcal{T}_{\widehat{\Gamma}}}([n],[m])\right)$ is contained in $\operatorname{Hom}_{\text {Aut }(\mathrm{V}, \times)}\left(\mathrm{V}^{\otimes n}, \mathrm{~V}^{\otimes m}\right)$, but here Aut $(\mathrm{V}, \times)$ is the special orthogonal group $\mathrm{SO}(\mathrm{V}, \mathrm{b})$.

Theorem 5.7. Let $\mathbb{F}$ be an infinite field of characteristic $\neq 2$, and let $\widehat{\Gamma}=$ $\left\{\widehat{\gamma}_{0}, \widehat{\gamma}_{1}, \widehat{\gamma}_{2}\right\}$, for $\widehat{\gamma}_{0}$ as in (5.6), $\widehat{\gamma}_{1}$ as in (5.3), and $\widehat{\gamma}_{2}$ as in (5.4). Then for any $n, m \in \mathbb{N}$,

$$
\mathcal{R}_{\widehat{\Gamma}}\left(\operatorname{Mor}_{\mathcal{T}_{\widehat{\Gamma}}}([n],[m])\right)=\operatorname{Hom}_{\mathrm{SO}(\mathrm{V}, \mathrm{b})}\left(\mathrm{V}^{\otimes n}, \mathrm{~V}^{\otimes m}\right) .
$$

Proof. It is enough to note that the classical invariant theory for $\mathrm{SO}(\mathrm{V}, \mathrm{b})$ (see Wey39] or [dCP76, Theorem 5.6]) shows that $\operatorname{Hom}_{\mathrm{SO}(\mathrm{V}, \mathrm{b})}\left(\mathrm{V}^{\otimes n}, \mathrm{~V}^{\otimes m}\right)$ is generated by composition and tensor products by $1_{\vee}, \mathrm{b}, \mathrm{b}^{t}, \tau$ and $\times$, because $\mathrm{b}(x \times y, z)$ is a determinant map on $\mathrm{V}$.

As in Section 4, we can get rid of circles and crossings in $\mathcal{T}_{\widehat{\Gamma}}$, and

$$
\Upsilon=0, \quad=-2
$$

Also, from $\widehat{\gamma}_{2}$ and the 3 -tangle obtained 'rotating it' we obtain:

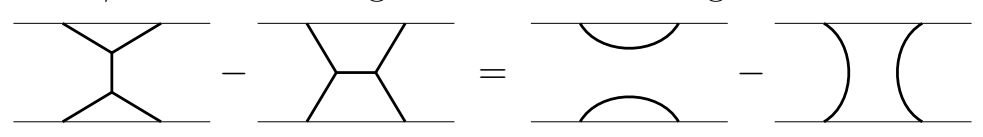


This allows us to replace cycles by linear combinations of 3-tangles without cycles. For instance:

$$
\begin{aligned}
& Y=\bar{Y}+Y-\bar{Q}=-Y
\end{aligned}
$$

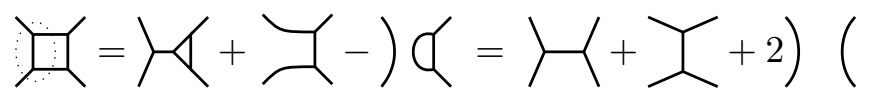

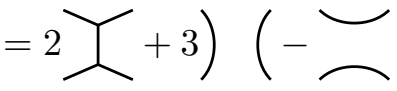

$$
\begin{aligned}
& \dot{\gamma}=\hat{R}+\hat{\gamma} \\
& =2 \gamma+\gamma+3\} \\
& Y=K+\underset{Y}{Y}=\cdots
\end{aligned}
$$

Therefore, any 3 -tangle is equivalent in $\mathcal{T}_{\widehat{\Gamma}}$ to a linear combination of 3 tangles without crossings, circles and cycles (i.e., a linear combination of planar trees). Let us call these tree 3-tangles.

Moreover, equation (5.8) can be transformed easily to

$$
Y-Y=Y_{-} Y^{\smile}
$$

that is,

$$
Y-Y=\begin{aligned}
& \text { linear combination of tree 3-tangles } \\
& \text { with a lower number of trivalent nodes. }
\end{aligned}
$$

and this gives a variation of associativity. For instance:

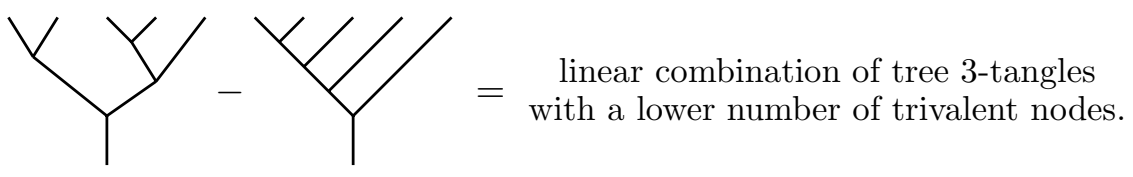

For any $n, m \in \mathbb{N}, n+m \geq 2$, the 3 -tangle $[n] \rightarrow[m]$ given by:

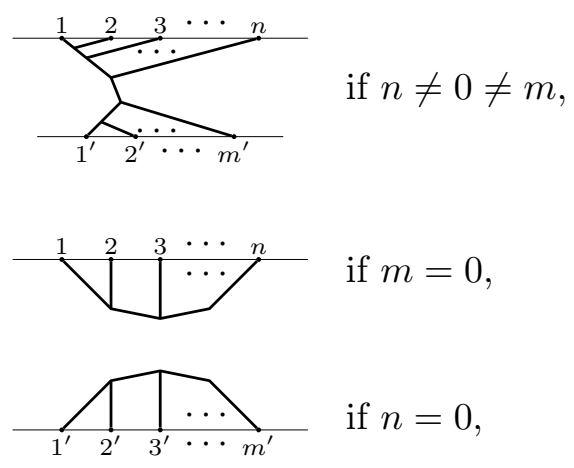

will be called the normalized connected 3 -tangle in $\operatorname{Mor}_{\mathcal{T}_{\widehat{\Gamma}}}([n],[m])$. The associativity above shows that any 3 -tangle in $\operatorname{Mor}_{\mathcal{T}_{\widehat{\Gamma}}}([n],[m])$ is a linear 
combination of 3-tangles which are a disjoint union of normalized connected 3-tangles. Disjoint unions of normalized connected 3-tangles will be called normalized 3 -tangles. They are determined by the 1 -valent vertices in each connected component. For instance, if $T:[6] \rightarrow[3]$ is a normalized 3 -tangle, $\partial T=\left\{1,2,3,4,5,6,1^{\prime}, 2^{\prime}, 3^{\prime}\right\}$, and the connected components split $\partial T$ into $\left\{1,2,3,6,3^{\prime}\right\},\{4,5\}$, and $\left\{1^{\prime}, 2^{\prime}\right\}$, then we have:

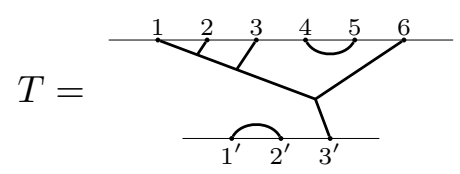

The number of normalized 3-tangles $[n] \rightarrow[m]$ is the number $a(n+m)$ of Catalan partitions of $n+m$, i.e., partitions of $n+m$ points around a circle into a disjoint union of subsets whose convex hulls are disjoint and such that all subsets have at least two elements. The sequence $a(n)$ is defined recursively by

$$
a(0)=1, a(1)=0, \quad a(n)=\frac{(n-1)(2 a(n-1)+3 a(n-2))}{n+1},
$$

and the terms $a(2 n)$ are the sequence \#A099251 in OEIS. The Catalan partition corresponding to the normalized 3-tangle above is pictured as follows:

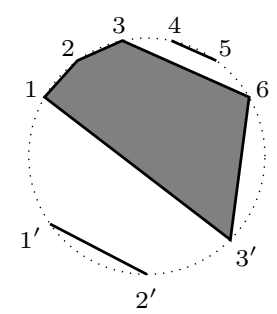

Theorem 5.12. Let $n, m \in \mathbb{N}$, and assume that the characteristic of $\mathbb{F}$ is 0 . Let $\mathfrak{V}=(\mathrm{V}, \mathrm{b}, \times)$ be a 3 -dimensional vector space $\mathrm{V}$ endowed with a nonzero cross product $x \times y$ relative to the nondegenerate symmetric bilinear form $\mathrm{b}$, and let $\widehat{\Gamma}=\left\{\widehat{\gamma}_{0}, \widehat{\gamma}_{1}, \widehat{\gamma}_{2}\right\}$, where $\widehat{\gamma}_{0}$ is as in (5.6), $\widehat{\gamma}_{1}$ as in (5.3), and $\widehat{\gamma}_{2}$ as in (5.4).

(a) The classes modulo $\widehat{\Gamma}$ of normalized 3 -tangles $[n] \rightarrow[\mathrm{m}]$ form a basis of $\operatorname{Mor}_{\mathcal{T}_{\widehat{\Gamma}}}([n],[m])$.

(b) $\mathcal{R}_{\widehat{\Gamma}}$ gives a linear isomorphism

$$
\operatorname{Mor}_{\mathcal{T}_{\widehat{\Gamma}}}([n],[m]) \rightarrow \operatorname{Hom}_{\mathrm{SO}(\mathrm{V}, \mathrm{b})}\left(\mathrm{V}^{\otimes n}, \mathrm{~V}^{\otimes m}\right) .
$$

(c) The normalized 3 -tangles $[n] \rightarrow[n]$ give a basis of the centralizer algebra

$$
\operatorname{End}_{\mathrm{SO}(\mathrm{V}, \mathrm{b})}\left(\mathrm{V}^{\otimes n}\right) \simeq \operatorname{Mor}_{\mathcal{T}_{\widehat{\Gamma}}}([n],[n])
$$

and $\operatorname{dim} \operatorname{End}_{\mathrm{SO}(\mathrm{V}, \mathrm{b})}\left(\mathrm{V}^{\otimes n}\right)$ equals the number a $(2 n)$ of Catalan partitions.

Proof. Extending scalars, we may assume that $\mathbb{F}$ is algebraically closed. Then $(\mathrm{V}, \mathrm{b})$ is isomorphic to the adjoint module $\mathfrak{s l}_{2}$ for the Lie algebra $\mathfrak{s l}_{2}$ of $2 \times 2$ matrices of trace 0 over $\mathbb{F}$, because $\mathfrak{s l}_{2}$ is the orthogonal Lie algebra relative to its Killing form, which is a nondegenerate symmetric bilinear 
form. Thus, $\operatorname{Hom}_{\mathrm{SO}(\mathrm{V}, \mathrm{b})}\left(\mathrm{V}^{\otimes n}, \mathbb{F}\right) \cong \operatorname{Hom}_{\mathfrak{s l}_{2}}\left(\mathfrak{s l}_{2}^{\otimes n}, \mathbb{F}\right)$, whose dimension also equals the number $a(n)$ of Catalan partitions. The same argument as in Theorem 4.10 now gives the result.

Remark 5.13. In particular, for $n=m$, Theorem 5.12 also gives a basis of the centralizer algebra $\operatorname{End}_{\mathfrak{s l}_{2}}\left(\mathfrak{s l}_{2}^{\otimes n}\right)$ of $\mathfrak{s l}_{2}$ over $\mathbb{F}$ acting on tensor powers of its adjoint module. Multiplication in this basis can be achieved as in Remark 4.16.

Remark 5.14. Relation $\widehat{\gamma}_{2}$ implies that $\operatorname{Mor}_{\mathcal{T}_{\widehat{\Gamma}}}([n],[0])$ is spanned by 3tangles where crossings are allowed, and where each connected component has at most one trivalent node. If there are two connected components with one trivalent node, we can proceed as follows, using $\widehat{\gamma}_{2}$ repeatedly:

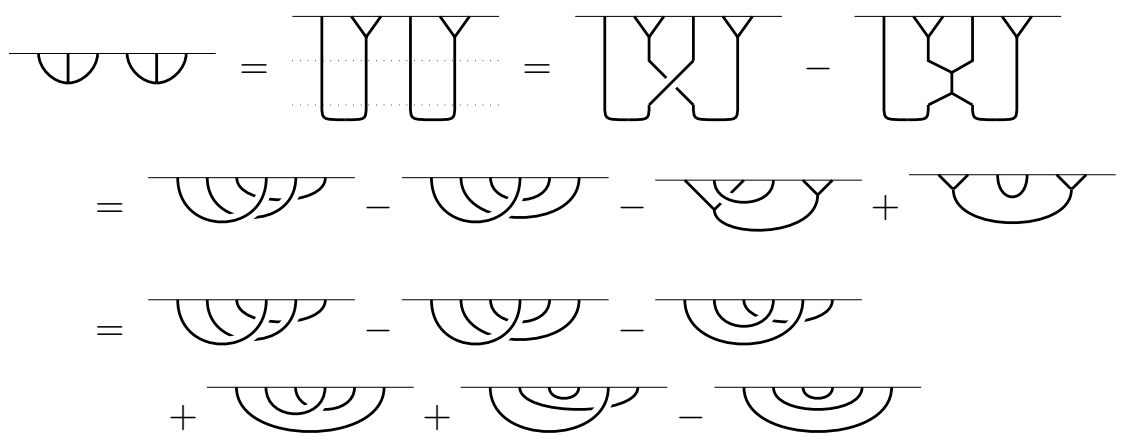

This corresponds, under $\mathcal{R}_{\mathcal{T}_{\widehat{\Gamma}}}$ to the identity

$$
\mathrm{b}\left(x_{1} \times x_{2}, x_{3}\right) \mathrm{b}\left(y_{1} \times y_{2}, y_{3}\right)=\operatorname{det}\left(\mathrm{b}\left(x_{i}, y_{j}\right)\right) .
$$

Hence, $\operatorname{Mor}_{\mathcal{T}_{\widehat{\Gamma}}}([n],[0])$ is spanned by 3-tangles, where there is at most one connected component with a trivalent node (and in this case $n$ must be odd). This reflects the fact that the algebra of invariants $\mathbb{F}[n \mathrm{~V}]^{\mathrm{SO}(\mathrm{V}, \mathrm{b})}$ for a 3-dimensional cross product algebra $\mathfrak{V}=(\mathrm{V}, \mathrm{b}, \times)$ is generated by the polynomial maps $\mathrm{b}\left(x_{i}, x_{j}\right)$ and $\operatorname{det}\left(x_{i}, x_{j}, x_{k}\right)=\mathrm{b}\left(x_{i} \times x_{j}, x_{k}\right)$, and, if $n$ is even, $\mathbb{F}[n \mathrm{~V}]^{\mathrm{SO}(\mathrm{V}, \mathrm{b})}=\mathbb{F}[n \mathrm{~V}]^{\mathrm{O}(\mathrm{V}, \mathrm{b})}$ is generated simply by the maps $\mathrm{b}\left(x_{i}, x_{j}\right)$, where $i, j, k \in\{1,2,3\}$. In particular, the isomorphism $\operatorname{Mor}_{\mathcal{T}_{\widehat{\Gamma}}}([n],[n]) \cong$ $\operatorname{Mor}_{\mathcal{T}_{\widehat{\Gamma}}}([2 n],[0])$ shows that the 3 -tangles can be folded to give 2-row Brauer algebra diagrams and reflects the fact that for $n=0,1, \ldots$, the centralizer algebra $\operatorname{End}_{\mathrm{SO}(\mathrm{V}, \mathrm{b})}\left(\mathrm{V}^{\otimes n}\right)=\operatorname{End}_{\mathrm{O}(\mathrm{V}, \mathrm{b})}\left(\mathrm{V}^{\otimes n}\right)$ is a homomorphic image of the Brauer algebra $\mathrm{B}_{n}(3)$. (Further details on Brauer algebras can be found in [Bra37].)

\section{A (1|2)-Dimensional CROSS PROduCt}

The 3-dimensional Kaplansky superalgebra over a field $\mathbb{F}$ of characteristic $\neq 2$ is a Jordan superalgebra $\mathrm{V}$ with one-dimensional even part $\mathrm{V}_{\overline{0}}=\mathbb{F} e$ and two-dimensional odd part $\mathrm{V}_{\overline{1}}=\mathbb{F} p \oplus \mathbb{F} q$, and with supercommutative multiplication given by:

$e \times e=e, e \times x=x \times e=\frac{1}{2} x \forall x \in \mathrm{V}_{\overline{1}}, p \times p=q \times q=0, p \times q=-q \times p=e$. 
For any two homogeneous elements $x, y \in \mathrm{V}$, the supercommutativity of the product gives $x \times y=(-1)^{x y} y \times x$, where $(-1)^{x y}$ is -1 if $x$ and $y$ are both odd, and it is 1 otherwise.

Consider the even nondegenerate supersymmetric bilinear form $\mathrm{b}: \mathrm{V} \times \mathrm{V} \rightarrow \mathbb{F}$ such that

$$
\mathrm{b}(e, e)=\frac{1}{2}, \quad \mathrm{~b}(p, q)=1 .
$$

The evenness of $b$ means $b\left(\bigvee_{\overline{0}}, \bigvee_{\overline{1}}\right)=0=b\left(\bigvee_{\overline{1}}, \bigvee_{\overline{0}}\right)$, and the supersymmetry means that $b$ is symmetric on $\bigvee_{\overline{0}}$ and skew symmetric on $V_{\overline{1}}$. Hence, equation (6.1) may be rewritten as

$$
e \times e=e, \quad e \times x=x \times e=\frac{1}{2} x, \quad x \times y=\mathrm{b}(x, y) e,
$$

for all $x, y \in \mathrm{V}_{\overline{1}}$.

Lemma 6.3. Let $\mathrm{V}$ be the 3-dimensional Kaplansky superalgebra. Assume $\left\{x_{1}, x_{2}, x_{3}\right\}$ and $\left\{y_{1}, y_{2}, y_{3}\right\}$ are dual bases of $\mathrm{V}$ relative to $\mathrm{b}\left(\right.$ i.e. $\mathrm{b}\left(x_{i}, y_{j}\right)=$ $\delta_{i, j}$ ) consisting of homogeneous elements.

(i) The form $\mathrm{b}$ is associative.

(ii) $\sum_{i=1}^{3} \mathrm{~b}\left(y_{i}, x_{i}\right)=-1$ and $\sum_{i=1}^{3} y_{i} \times x_{i}=0$.

(iii) For any homogeneous elements $z_{1}, z_{2}, z_{3}, z_{4} \in \mathrm{V}$,

$$
\begin{aligned}
\mathrm{b}\left(z_{1} \times z_{2}, z_{3} \times z_{4}\right)= & \mathrm{b}\left(z_{1}, z_{2}\right) \mathrm{b}\left(z_{3}, z_{4}\right) \\
& +(-1)^{z_{2} z_{3}} \frac{1}{2} \mathrm{~b}\left(z_{1}, z_{3}\right) \mathrm{b}\left(z_{2}, z_{4}\right)+\frac{1}{2} \mathrm{~b}\left(z_{1}, z_{4}\right) \mathrm{b}\left(z_{2}, z_{3}\right) .
\end{aligned}
$$

(Note that $\mathrm{b}\left(z_{1}, z_{4}\right) \mathrm{b}\left(z_{2}, z_{3}\right)=(-1)^{z_{4} z_{2}}(-1)^{z_{4} z_{3}} \mathrm{~b}\left(z_{1}, z_{4}\right) \mathrm{b}\left(z_{2}, z_{3}\right)$, because $\mathrm{b}\left(z_{2}, z_{3}\right)=0$ unless $z_{2}$ and $z_{3}$ have the same parity.)

Proof. The expressions $\sum_{i=1}^{3} \mathrm{~b}\left(y_{i}, x_{i}\right)$ and $\sum_{i=1}^{3} y_{i} \times x_{i}$ do not depend on the dual bases chosen, so we may take $x_{1}=e=\frac{1}{2} y_{1}, x_{2}=p=-y_{3}, x_{3}=q=y_{2}$. The result now follows by straightforward computations.

Consider the triple $\mathfrak{V}=(\mathrm{V}, \mathrm{b}, \times)$, but now denote by $\tau$ the super-switch map: $\tau: \mathrm{V}^{\otimes 2} \rightarrow \mathrm{V}^{\otimes 2}, x \otimes y \mapsto(-1)^{x y} y \otimes x$, for any homogeneous elements $x, y \in \mathrm{V}$. As in Theorem 3.8, there is a unique functor $\mathcal{R}_{\mathfrak{V}}: \mathcal{T} \rightarrow \mathcal{V}$ with the same properties expressed there (but taking into account that now $\tau$ is the super-switch map).

Consider also the following linear maps (where $\mathrm{m}(x \otimes y)=x \times y$ ):

$$
\begin{aligned}
& \mathrm{c}_{0}^{\mathrm{s}} \in \operatorname{Hom}_{\mathbb{F}}\left(\mathrm{V}^{\otimes 0}, \mathrm{~V}^{\otimes 0}\right): 1 \mapsto\left(\mathrm{b} \circ \mathrm{b}^{t}\right)(1)+1 \text {, } \\
& \mathrm{c}_{1}^{\mathrm{s}} \in \mathrm{Hom}_{\mathbb{F}}\left(\mathrm{V}^{\otimes 2}, \mathrm{~V}\right): x \otimes y \mapsto(1-\tau) \circ \mathrm{m}(x \otimes y)=x \times y-(-1)^{x y} y \times x, \\
& c_{2}^{\mathrm{s}} \in \operatorname{Hom}_{\mathbb{F}}\left(\mathrm{V}^{\otimes 3}, \mathrm{~V}\right): x \otimes y \otimes z \mapsto(x \times y) \times z-\mathrm{b}(x, y) z \\
& +(-1)^{y z} \frac{1}{2} \mathrm{~b}(x, z) y+\frac{1}{2} \mathrm{~b}(y, z) x \\
& c_{3}^{\mathrm{s}} \in \operatorname{Hom}_{\mathbb{F}}(\mathbb{F}, \mathrm{V}): 1 \mapsto\left(\mathrm{m} \circ \mathrm{b}^{t}\right)(1),
\end{aligned}
$$

for homogeneous elements $x, y, z \in \mathrm{V}$. The supercommutativity of $\mathrm{V}$ shows that $c_{1}^{s}=0$, and Lemma 6.3 and the nondegeneracy of $b$ prove that $c_{0}^{s}=0$, $c_{2}^{\mathfrak{s}}=0$, and $c_{3}^{\mathfrak{s}}=0$. 
The linear maps $c_{0}^{s}, c_{1}^{s}, c_{2}^{s}$, and $c_{3}^{s}$, are the images under $\mathcal{R}_{\mathfrak{V}}$ of:

$$
\gamma_{1}^{\mathrm{s}}=\gamma^{\mathrm{s}}=\Upsilon_{-1}^{+1}
$$

Instead of $\tilde{\gamma}_{2}^{\mathrm{s}}$, let us consider the element $\gamma_{2}^{\mathrm{s}} \in \operatorname{Mor}_{\mathcal{T}_{2}}([2],[2])$ given by $\gamma_{2}^{\mathrm{s}}=\left(\tilde{\gamma}_{2}^{\mathrm{s}} \sqcup \mathrm{I}_{1}\right) \circ\left(\mathrm{I}_{2} \sqcup \beta^{t}\right)$ :

$$
\gamma_{2}^{\mathrm{s}}=\nearrow^{\nearrow}-\frac{1}{2} \searrow\left(-\frac{1}{2}\right)(
$$

As in Corollary 3.10, if $\Gamma^{\mathrm{s}}=\left\{\gamma_{0}^{\mathrm{s}}, \gamma_{1}^{\mathbf{s}}, \gamma_{2}^{\mathbf{s}}, \gamma_{3}^{\mathbf{s}}\right\}$, there is a unique functor $\mathcal{R}_{\Gamma^{\mathrm{s}}}: \mathcal{T}_{\Gamma^{\mathrm{s}}} \rightarrow \mathcal{V}$ such that $\mathcal{R}_{\mathcal{V}}=\mathcal{R}_{\Gamma^{\mathrm{s}}} \circ \mathcal{P}$, with $\mathcal{P}$ the natural projection $\mathcal{T} \rightarrow \mathcal{T}_{\Gamma^{\mathrm{s}}}$.

The Lie superalgebra of derivations of $(\mathrm{V}, \times)$ is the orthosymplectic superalgebra $\mathfrak{o s p}(\mathrm{V}, \mathrm{b}) \simeq \mathfrak{o s p}_{1 \mid 2}$ over $\mathbb{F}$, and hence, $\mathcal{R}_{\Gamma^{\mathrm{s}}}\left(\operatorname{Mor}_{\mathcal{T}_{\Gamma^{\mathrm{S}}}}([n],[m])\right)$ is contained in $\operatorname{Hom}_{\mathfrak{o s p}(\mathrm{V}, \mathrm{b})}\left(\mathrm{V}^{\otimes n}, \mathrm{~V}^{\otimes m}\right)$.

By the arguments of Section $5, \gamma_{0}^{\mathrm{s}}$ allows us to get rid of circles and $\gamma_{2}^{\mathrm{s}}$ of crossings. Also, $\gamma_{2}^{\mathrm{s}}$ gives:

$$
\begin{aligned}
\Upsilon & =\widetilde{C}=C^{\prime} \Upsilon^{\prime}+\frac{1}{2} \bigcirc \mid \\
& =\left(1+\frac{1}{2}-\frac{1}{2}\right)|=|
\end{aligned}
$$

Now from $\gamma_{2}^{\mathrm{s}}$ and the 3-tangle obtained rotating it, we get the following relation in $\mathcal{T}_{\Gamma^{\mathrm{s}}}$ :

$$
\gamma-\gamma=\frac{1}{2}\left[\Upsilon^{-}\right)(]
$$

(compare with (5.8)), and this allows us, as in Section 5 , to replace cycles by linear combinations of 3-tangles without crossings, circles and cycles, and eventually to express any 3 -tangle in $\mathcal{T}_{\Gamma^{\mathrm{s}}}$ as a linear combination of 3-tangles which are disjoint unions of normalized connected 3-tangles.

Theorem 6.7. Let $\mathrm{V}$ be the 3-dimensional Kaplansky superalgebra over a field $\mathbb{F}$ of characteristic 0 , and assume $\mathrm{b}$ is the nondegenerate supersymmetric bilinear form on $\mathrm{V}$. Let $n, m \in \mathbb{N}$. Assume $\Gamma^{\mathrm{s}}=\left\{\gamma_{0}^{\mathrm{s}}, \gamma_{1}^{\mathrm{s}}, \gamma_{2}^{\mathrm{s}}, \gamma_{3}^{\mathrm{s}}\right\}$, where these 3 -tangles are as in (6.4) and (6.5). 
(a) The classes modulo $\Gamma^{\mathbf{s}}$ of the normalized 3-tangles $[n] \rightarrow[\mathrm{m}]$ form a basis of $\operatorname{Mor}_{T_{\Gamma^{s}}}([n],[m])$.

(b) The functor $\mathcal{R}_{\Gamma^{\mathrm{s}}}$ gives a linear isomorphism

$$
\operatorname{Mor}_{\mathcal{T}_{\Gamma^{\mathrm{s}}}}([n],[m]) \rightarrow \operatorname{Hom}_{\mathfrak{o s p}}(\mathrm{V}, \mathrm{b})\left(\mathrm{V}^{\otimes n}, \mathrm{~V}^{\otimes m}\right) \text {. }
$$

(c) The normalized 3-tangles $[n] \rightarrow[n]$ give a basis of the centralizer algebra

$$
\operatorname{End}_{\mathfrak{o s p}(\mathrm{V}, \mathrm{b})}\left(\mathrm{V}^{\otimes n}\right) \simeq \operatorname{Mor}_{\mathcal{T}_{\Gamma^{\mathrm{s}}}}([n],[n]),
$$

and $\operatorname{dim} \operatorname{End}_{\mathfrak{o s p}(\mathrm{V}, \mathrm{b})}\left(\mathrm{V}^{\otimes n}\right)$ equals the number $a(2 n)$ of Catalan partitions.

Proof. Extending scalars, we may assume that $\mathbb{F}$ is algebraically closed.

Given any unital commutative associative superalgebra $R=R_{\overline{0}} \oplus R_{\overline{1}}$ over $\mathbb{F}$, let $\alpha \in R_{\overline{0}}, \beta, \gamma \in R_{\overline{1}}$, and consider the even element $u=\alpha e+\beta p+\gamma q$ in the scalar extension $\mathrm{V} \otimes_{\mathbb{F}} R$. Then $\mathrm{b}(u, u)=\frac{1}{2} \alpha^{2}+2 \beta \gamma$, so

$$
\mathrm{b}(u, u)^{3}=\frac{1}{8}\left(\alpha^{6}+12 \alpha^{4} \beta \gamma\right)
$$

because $\beta^{2}=\gamma^{2}=0$, and

$$
\begin{aligned}
\mathrm{b}(u \times u, u) & =\mathrm{b}\left(\left(\alpha^{2}+2 \beta \gamma\right) e+\alpha \beta p+\alpha \gamma q, \alpha e+\beta p+\gamma q\right) \\
& =\frac{1}{2}\left(\alpha^{2}+2 \beta \gamma\right) \alpha+2 \alpha \beta \gamma=\frac{1}{2}\left(\alpha^{3}+6 \alpha \beta \gamma\right),
\end{aligned}
$$

and hence we obtain

$$
\mathrm{b}(u \times u, u)^{2}=\frac{1}{4}\left(\alpha^{6}+12 \alpha^{4} \beta \gamma\right) .
$$

This means that, up to scalars, $\mathrm{b}(u \times u, u)$ is the super Pfaffian (see [Ser92]). The First Fundamental Theorem of Invariant Theory for the special orthosymplectic group $\operatorname{SOSp}(\mathrm{V}, \mathrm{b})$ [LZ15] shows that $\mathcal{R}_{\Gamma^{\mathrm{s}}}$ gives a surjection

$$
\operatorname{Mor}_{\mathcal{T}_{\Gamma^{\mathrm{s}}}}([n],[m]) \rightarrow \operatorname{Hom}_{\mathrm{SOSp}(\mathrm{V}, \mathrm{b})}\left(\mathrm{V}^{\otimes n}, \mathrm{~V}^{\otimes m}\right)=\operatorname{Hom}_{\mathfrak{o s p}}(\mathrm{V}, \mathrm{b})\left(\mathrm{V}^{\otimes n}, \mathrm{~V}^{\otimes m}\right) .
$$

Now it follows from RS82 that the dimension of $\operatorname{Hom}_{\mathfrak{o s p}}(\mathrm{V}, \mathrm{b})\left(\mathrm{V}^{\otimes n}, \mathrm{~V}^{\otimes m}\right)$ coincides with the dimension of $\operatorname{Hom}_{\mathfrak{s l}_{2}}\left(\mathfrak{s l}_{2}^{\otimes n}, \mathfrak{s l}_{2}^{\otimes m}\right)$ for the adjoint module for $\mathfrak{s l}_{2} \simeq \mathfrak{s o}_{3}$, and this dimension equals the number of Catalan partitions of $n+m$, that is, the number of normalized 3-tangles $[n] \rightarrow[m]$ (see the comments before Theorem (5.12). Hence, the result holds.

Example 6.8. Let us consider explicitly the basis of the centralizer algebra $\operatorname{End}_{\mathfrak{o s p}(\mathrm{V}, \mathrm{b})}\left(\mathrm{V}^{\otimes 3}\right)$ or, equivalently, of $\operatorname{Mor}_{\mathcal{T}_{\Gamma^{\mathrm{s}}}}([3],[3])$. As in Remark 5.14, this is a homomorphic image of the Brauer algebra $B_{3}(-1)$ due to tangle relation $\gamma_{0}^{\mathbf{s}}$ (in fact, it is isomorphic to $B_{3}(-1)$ ), but here a basis different from the standard diagram basis of the Brauer algebra is obtained. According to Theorem 6.7, a basis of $\operatorname{Mor}_{\mathcal{T}_{\Gamma^{s}}}([3],[3])$ is given by the classes modulo $\Gamma^{\mathrm{s}}$ of the following 3 -tangles:
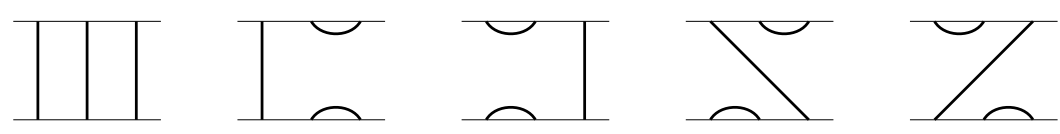


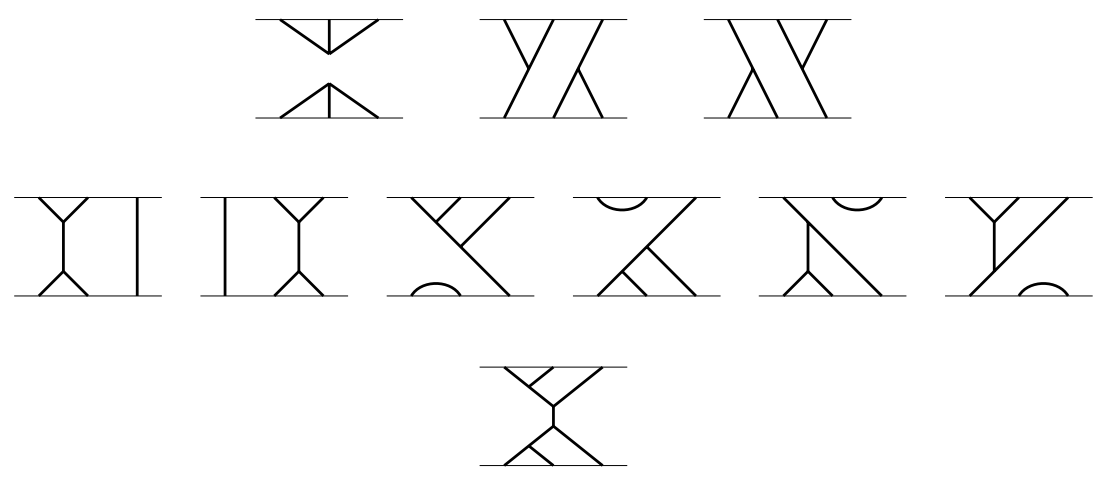

It is straightforward to translate this basis of normalized 3-tangles to a basis of the centralizer algebra $\operatorname{End}_{\mathfrak{o s p}(\mathrm{V}, \mathrm{b})}\left(\mathrm{V}^{\otimes 3}\right)$. For example,

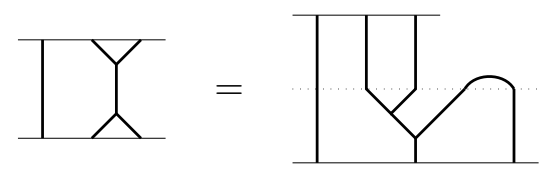

and hence, if $\left\{x_{1}, x_{2}, x_{3}\right\}$ and $\left\{y_{1}, y_{2}, y_{3}\right\}$ are two dual bases of $\mathbf{V}$ consisting of homogeneous elements, then using Proposition 2.5, which is valid in the super setting, we obtain that the corresponding element of the centralizer is the composition

$$
u \otimes v \otimes w \mapsto \sum_{i=1}^{3} u \otimes v \otimes w \otimes y_{i} \otimes x_{i} \mapsto \sum_{i=1}^{3} u \otimes\left((v \times w) \times y_{i}\right) \otimes x_{i} .
$$

In the same vein,

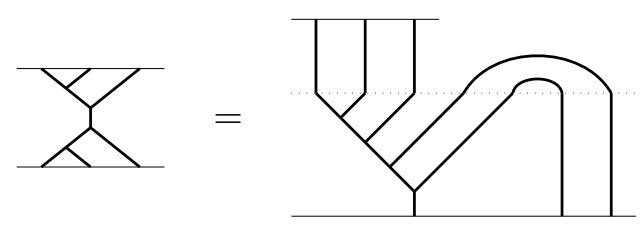

which corresponds to the map

$$
u \otimes v \otimes w \mapsto \sum_{i, j=1}^{3}\left(\left(((u \times v) \times w) \times y_{i}\right) \times y_{j}\right) \otimes x_{j} \otimes x_{i} .
$$

\section{REFERENCES}

[Boo98] D. Boos, Ein tensorkategorieller Zugang zum Satz von Hurwitz, Diplomarbeit, Regensburg und Zürich, 1998. http://www.mathematik. uni-bielefeld.de/r̃ost/tensors.html\# boos

[Bra37] R. Brauer, On algebras which are connected with semisimple Lie groups, Annals of Math. 38 (1937), 857-872.

[BG67] R.B. Brown and A. Gray, Vector cross products, Comment. Math. Helv. 42 (1967), 222-236.

[CKR05] L. Cadorin, M.-A. Knus, and M. Rost, On the dimension and other numerical invariants of algebras and vector products, Algebra and Number Theory, 34-65, Hindustan Book Agency, Delhi, 2005.

[dCP76] C. de Concini and C. Procesi, A characteristic free approach to invariant theory, Advances in Math. 21 (1976), no. 3, 330-354. 
[Kup94] G. Kuperberg, The quantum $G_{2}$ link invariant. Internat. J. Math. 5 (1994), no. $1,61-85$.

[Kup96] G. Kuperberg, Spiders for rank 2 Lie algebras, Comm. Math. Phys. 180 (1996), no. 1, 109-151.

[LZ15] G.I. Lehrer and R.B. Zhang, Invariants of the orthosymplectic Lie superalgebra and super Pfaffians, arXiv:1507.01329.

[OEIS] N. J. A. Sloane, The on-line encyclopedia of integer sequences (OEIS) 2010, http://oeis.org.

[RS82] V. Rittenberg and M. Scheunert, A remarkable connection between the representations of the Lie superalgebras osp $(1,2 n)$ and the Lie algebras o $(2 n+1)$, Comm. Math. Phys. 83 (1982), no. 1, 1-9.

[Ros04] M. Rost, On vector product algebras, https://www.math.uni-bielefeld.de/r̃ost/data/vpg.pdf

[Scha66] R.D. Schafer, An Introduction to Nonassociative Algebras, Pure and Applied Mathematics, Vol. 22. Academic Press, New York-London 1966.

[Schw88] G.W. Schwarz, Invariant theory of $G_{2}$ and $\operatorname{Spin}_{7}$, Comment. Math. Helv. 63 (1988), no. 4, 624-663.

[Ser92] A.N. Sergeev, An analogue of the classical theory of invariants for Lie superalgebras, (Russian) Funktsional. Anal. i Prilozhen. 26 (1992), no. 3, 88-90; translation in Funct. Anal. Appl. 26 (1992), no. 3, 223-225.

[Wes07] B.W. Westbury, Enumeration of non-positive planar trivalent graphs, J. Algebraic Combin. 25 (2007), no. 4, 357-373.

[Wey39] H. Weyl, The Classical Groups. Their Invariants and Representations, Princeton University Press, Princeton, N.J., 1939.

[ZSh15] A.N. Zubkov and I.P. Shestakov, Invariants of $G_{2}$ and $\operatorname{Spin}_{7}$ in positive characteristic, arXiv:1512.06354.

Department of Mathematics, University of Wisconsin-Madison, Madison, WI 53706, USA

E-mail address: benkart@math.wisc.edu

Departamento de Matemáticas e Instituto Universitario de Matemáticas y Aplicaciones, Universidad de Zaragoza, 50009 Zaragoza, Spain

E-mail address: elduque@unizar.es 